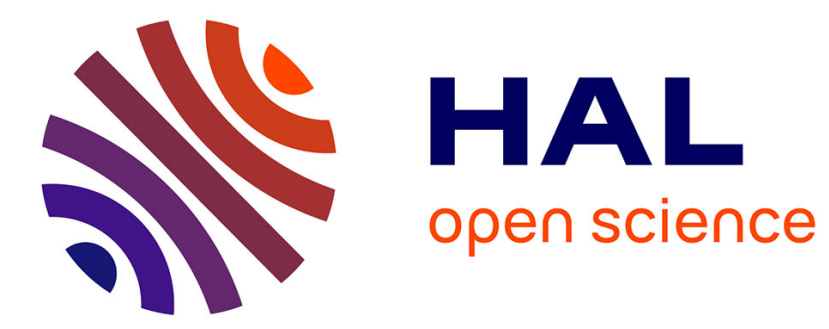

\title{
Mai 68 et ses suites en géographie française Olivier Orain
}

\section{To cite this version:}

Olivier Orain. Mai 68 et ses suites en géographie française. Revue d'histoire des sciences humaines, 2015, Les "années 68" des sciences humaines et sociales, 26, pp.209-242. 10.4000/rhsh.2406 . halshs01120325

\section{HAL Id: halshs-01120325 https://shs.hal.science/halshs-01120325}

Submitted on 13 Feb 2018

HAL is a multi-disciplinary open access archive for the deposit and dissemination of scientific research documents, whether they are published or not. The documents may come from teaching and research institutions in France or abroad, or from public or private research centers.
L'archive ouverte pluridisciplinaire HAL, est destinée au dépôt et à la diffusion de documents scientifiques de niveau recherche, publiés ou non, émanant des établissements d'enseignement et de recherche français ou étrangers, des laboratoires publics ou privés. 


\title{
Mai 68 et ses suites en géographie française
}

\author{
Olivier Orain \\ Chargé de recherche au CNRS (UMR 8504 Géographie-cités) \\ Équipe Épistémologie et histoire de la géographie (EHGO)
}

Résumé Cet article fait le point sur les évolutions qu'a connues la géographie française durant les décennies 1960 et 1970 et met l'accent sur les transformations politico-syndicales et épistémologiques survenues, immédiatement ou avec un certain délai, à l'issue des événements de 68. II met en lumière dans un premier temps le relatif consensus et I'homogénéité d'une corporation dont l'expansion démographique dans l'après-guerre s'est faite sans transformation majeure des manières de faire ou des équilibres internes. II explore ensuite la trajectoire des instituts et sections de géographie durant le mouvement de maijuillet 1968, qui à la fois participent à la dynamique contestataire générale et réinventent leur fonctionnement interne, tout en voulant réformer dans un sens assez légitimiste les contenus d'enseignement. Enfin, il indique comment des processus de clivage interviennent essentiellement après la crise elle-même : politiques d'abord, avec l'émergence et la cristallisation d'une droite géographique jusque-là évanescente; scientifique ensuite, avec le développement du processus contestataire souvent appelé " nouvelle géographie ».

Mots-clés : histoire de la géographie, France, nouvelle géographie, Mai 68

Abstract This article summarizes what is actually well-known about the evolution of French geography during the 60's and the 70's and then focuses on political and epistemological changes that appeared immediately or following in wake of 1968 's events. It first shows how the geographic community more or less maintained social and political consensus and homogeneity through its demographic expansion during post-war decades, which price was an epistemological conservatism and a static equilibrium between old constituents of the discipline. Then it depicts how Departments of Geography in universities were mobilized during the months of contestation (May-July 1968), mixing a global involvement within the national movement and a local reform of geographical communities and institutions, that shows a conformist view of what geography is, similar to common idiosyncratic self-representations mastered by geographic leaders. Lastly, it explores the political break-ups that immediately followed the 1968 crisis and the more delayed rise of a scientific criticism called "nouvelle géographie" and partly inspired by the English-American "new geography", but specific in the way it mixes political and epistemological dimensions.

Keywords : History of Geography, France, New Geography, May 68 
On comprend que sociologues et géographes se soient si clairement dis-
tingués, au sein du mouvement de contestation de I'Université, au point
de symboliser, notamment dans le mouvement syndical, l'opposition entre
la tendance « gauchiste " et la tendance " réformiste ", entre la contes-
tation globale et « radicale » de l'institution universitaire et du monde
social et la revendication « corporatiste » mettant l'accent sur les carrières
des enseignants ou la transformation des méthodes et des contenus de
l'enseignement.
Pierre Bourdieu, Homo Academicus, Paris, Minuit, 1984, p. 224.

Un contraste important existe entre les traces publiques fort ténues de la participation des géographes français aux événements de Mai 68 et une mémoire collective, à usage interne, qui en a fait un moment décisif de schisme au sein d'une communauté décrite comme auparavant très homogène et unie. II y va sans doute de la discrétion et de la faible exposition d'une communauté disciplinaire produisant davantage d'administrateurs et de spécialistes que d'intellectuels médiatiques - les mieux placés pour rendre exemplaire et notoire une appartenance savante. Elle apparaît néanmoins, et de manière significative, dans une comparaison de Pierre Bourdieu dans Homo Academicus dont j'ai reproduit en exergue les éléments de conclusion. Aux sociologues, parangons de la contestation politique, aurait alors échu un répertoire universel, dépassant la seule crise universitaire; aux géographes des revendications strictement catégorielles, peu propices à une montée en généralité révolutionnaire, posture lue comme la résultante de "l'humilité des dispositions qui conviennent à leurs positions » (Bourdieu, 1984, 45) « situé[e]s au niveau le plus bas des hiérarchies tant sociales que scolaires » (ibid., 223).

Point d'orgue d'un regard souvent critique et à tout le moins décapant sur les géographes et leurs mœurs ${ }^{1}$, Homo Academicus a scandalisé ceux qui parmi ces derniers n'ont pas saisi l'ambivalence de l'analyse et l'attention qui l'a rendue possible. Il n'est pas question dans les pages qui viennent de me donner pour but de corroborer ou d'infirmer une appréciation tributaire de modalités d'expression (l'arène du « mouvement syndical ", les défilés communs) largement aveugle à ces temps d'entre-soi qui auraient permis de la nuancer. Par contraste, il m'est apparu souhaitable de sortir de la confidentialité la masse d'informations et de bilans informels qui existent à propos de cette période critique et d'en présenter une synthèse provisoire, néanmoins avertie par la lecture bourdieusienne (quitte à la relativiser) et formant un cadre pour des approfondissements ultérieurs. Au demeurant, l'enjeu de fond est moins d'expliquer une posture supposée typique des géographes durant la séquence courte des « événements " que de réfléchir à l'incidence de ces derniers à l'entame d'une crise savante qui a profondément modifié la physionomie d'une discipline marquée durant les soixantedix années précédentes par un consensus rarement troublé.

1 |l faut signaler la contribution décisive aux analyses de Pierre Bourdieu qu'ont représentées les enquêtes sur les étudiants menées dans les années 1960 par Noëlle Bisseret, dont témoigne particulièrement I'article « La sélection à l'université et sa signification pour l'étude des rapports de dominance » (Bisseret, 1968). 
Pour des raisons aisément compréhensibles, la dimension mémorielle occupe une place centrale dans cette synthèse ${ }^{2}$ : nombre d'archives sont encore à inventorier, car éparpillées entre les différents instituts ou départements de géographie qui maillent le territoire universitaire français, sans parler de ce qui a été probablement perdu ${ }^{3}$. Par contraste, les géographes qui ont été témoins ou acteurs à l'époque s'expriment volontiers sur leur expérience, manifestant parfois à cette occasion un métier très sûr de social scientist, significatif des mutations qu'a connues l'habitus professionnel depuis cette époque de la fin des années 1960. Un matériau original a été recueilli à l'occasion d'entretiens, de conversations informelles et de réunions dédiées ${ }^{4}$. Les manuels historiographiques publiés à partir du milieu des années $1990^{5}$ - quand les programmes du CAPES et de l'agrégation de géographie se sont ouverts à l'histoire et à l'épistémologie disciplinaire - sont eux aussi fondés sur une dimension testimoniale pour ce qui concerne la période : leur évocation des « années 68 " s'appuie implicitement sur l'expérience d'auteurs qui en ont été acteur ou spectateur. En ce sens, ils font matière commune avec le genre " égogéographie ", de format et d'intention fort variables, qui a émergé à la même période ${ }^{6}$.

J'ai utilisé en contrepoint les fascicules du Bulletin de liaison des instituts et centres de recherche de géographie, plus communément appelé Intergéo, que Jean Dresch? a créé en 1966. Cet inventaire minutieux des lieux et des pratiques en tous genres de la géographie française, en dépit de son caractère généralement neutre et de ses lacunes inévitables, fournit des informations précieuses sur une large gamme de sujets, notamment via ses comptes rendus des réunions des divers comités patronnant la géographie française ou la reproduction d'enquêtes et de rapports. Le poids important de ces archives institutionnelles contraste avec le peu d'éléments disponibles sur le mouvement étudiant. J'ai exploité des comptes rendus d'AG, tracts, libelles et autres

2 Je tiens à remercier Nicolas Ginsburger et Marie-Claire Robic pour leur relecture attentive du texte initial et leurs remarques.

3 En 1977, décision a été prise à l'institut de géographie de Paris de mettre au pilon les « documents en très mauvais état de conservation entreposés dans la réserve de la Bibliothèque » (arrêté du 20 janvier 1977). Les archives bordelaises semblent également perdues.

4 En particulier une matinée de débat au sein du comité de rédaction de la revue L'Espace géographique, le vendredi 16 mai 2008, qui fut accompagnée de contributions écrites proposées par Benoît Antheaume, Claude Bataillon et François Durand-Dastès (le tout inédit à ce jour). J'en profite pour adresser mes plus sincères remerciements aux nombreux collègues qui se sont exprimés à cette occasion ou que j'ai interviewés depuis.

5 On citera notamment Scheibling, 1994; Marconis, 1996; Claval, 1998.

6 Dans le cas de Paul Claval, la proximité est particulièrement marquée : deux ans séparent La géographie comme genre de vie. Un itinéraire intellectuel (1996) et Histoire de la géographie de 1870 à nos jours (1998), qui comporte de nombreux développements autobiographiques, même si ce n'est pas le cas à propos de Mai 1968.

7 Né en 1905, normalien, géomorphologue, élève d’Emmanuel de Martonne, "intellectuel communiste engagé » (J.-L. Tissier) durant la quasi-totalité de sa carrière, il est dans les années 1960 l'un des principaux patrons de la géographie française, directeur de l'Institut de géographie de Paris et du Centre de documentation cartographique et géographique (CRDCG), président du Comité national français de géographie, président de la section de géographie du CNRS entre 1965 et 1969, codirecteur des Annales de géographie, etc. 
formes d'expression spontanée, qui renvoient une image beaucoup moins consensuelle et policée des confrontations de $1968^{\circ}$. À l'exception des personnes qui ont fait carrière dans l'université, il est en revanche assez difficile de retrouver ces acteurs, peu visibles individuellement dans les documents d'époque et dispersés par la suite.

Quel que puisse être ma sensibilité relative à cette époque ${ }^{9}$, j'ai essayé de conserver aux éléments de tableau ou de récit qui suivent un horizon d'impartialité et de neutralité axiologique, afin de contrebalancer le point de vue spontané que j'aurais pu adopter. J'ai été aidé pour ce faire par les récits qui ont été publiés (Saunier-Seité, 1982; Claval, 1996 et 1998; Demangeot dans Daudel, 2008) ou que j'ai pu recueillir. Toute la difficulté d'une écriture de la séquence réside dans le choix d'une présentation symétrique de positions qui se polarisent alors fortement et ont pu demeurer antagonistes sur plusieurs décennies, renforcées sinon alimentées par divers épisodes ultérieurs. Je voudrais en effet monter que les effets clivants de la crise de mai-juin 68 ont été particulièrement forts dans une communauté disciplinaire pour laquelle les carrières universitaires représentaient une opportunité d'ascension sociale. Une polarisation socioscientifique s'est développée autour de l'adhésion à ou du rejet d'une tradition porteuse d'un système de valeurs socioscientifiques héritées, classant dès lors de façon tranchée les individus et les initiatives. J'ai construit mon propos sur le mode de la « traversée » dont j'ai dit le pouvoir régulateur dans mon bilan historiographique "Une fertilisation paradoxale ", dans ce même volume. Le tableau qui ouvre cette présentation fait état des transformations mais aussi des inerties qui caractérisent la communauté géographique dans la période précédant mai-juin 1968. II s'agit ensuite d'analyser à partir des exemples les mieux documentés les processus de confrontation et d'enrôlement qui s'accusent entre mai 1968 et l'automne 1970, moment où la solution d'une scission des corps se concrétise et prend l'avantage. Enfin, la question de l'incidence - directe ou symbolique - de cette fracture a priori sociopolitique est posée, notamment au travers de ce qui ressemble en apparence à une traduction (au sens de déplacement) épistémologique.

\section{La fin d'une époque?}

La géographie française a connu durant les décennies d'après-guerre une expansion assez conforme à l'évolution générale de l'université française (Robic, 1989). Son cursus sans latin et son positionnement entre sciences et lettres en a fait une voie attractive pour les élèves des filières modernes du secondaire ${ }^{10}$, avec pour corrélat un

8 On en trouvera un exemple significatif (encore tardif) avec le document « Propositions destructives » figurant dans ce dossier.

9 Enfant de "soixante-huitards » durablement étiquetés tels des décennies durant, j'ai conscience d'avoir une position non neutre sur ce sujet, renforcée par ma socialisation spécifique au sein de la géographie.

10 Ainsi que le confirment notamment les enquêtes de Noëlle Bisseret (Bisseret, 1968). 
recrutement davantage populaire et provincial que dans la plupart des autres filières des facultés de lettres. Ces caractéristiques se retrouvent au niveau du personnel enseignant, recruté à tour de bras durant les années de forte demande (1956-1972) au sortir des épreuves de l'agrégation ou, en moindre mesure, dans les viviers locaux. Peu attractive alors pour les normaliens de la rue d'Ulm, la carrière de géographe I'est davantage pour ceux de Saint-Cloud (Tissier, 1985), dont les origines sociales sont proches de celles de leurs homologues non-normaliens, voire plus modestes encore. Si les métiers de l'enseignement constituent le marché privilégié d'une formation à la discipline fortement tributaire d'un horizon scolaire, les " géographes professionnels » (cartographes, aménageurs, techniciens, fonctionnaires territoriaux) ont pris progressivement de l'importance, initialement au service de l'animation économique régionale, puis durant les années 1960 dans des missions diverses associées à la promotion de la " géographie appliquée ", en particulier dans l'aménagement du territoire. Ce marché nouveau a également fourni des responsabilités éminentes à plusieurs universitaires proches des cercles gaullistes, Jacqueline Beaujeu-Garnier et Jean Labasse en particulier.

Pourtant, opportunités nouvelles et missions prestigieuses masquent difficilement la position structurellement dominée des géographes sur cette nouvelle scène et le sentiment de rivalité ressenti à l'endroit de "concurrents » divers, ingénieurs, économistes, sociologues. Les postures théoriques et les conceptions de l'intervention publique de ces derniers constituaient un défi et un repoussoir pour une corporation partagée entre complexe obsidional et malaise diffus ". La découverte progressive de la raison scientifico-ingénieuriale de l'époque, prégnante dans les milieux de la planification et de l'aménagement, y compris dans sa déclinaison en sciences sociales, a principalement incité les géographes à un contre-pied mettant en valeur le sens des " réalités », de la complexité et de la diversité infinie des phénomènes supposé intrinsèque à leur " démarche ». À longueur d'articles et d'ouvrages pourchassant l'identité disciplinaire, la production "réflexive » des années 1960 redit inlassablement une doxa identitaire dont le porte-à-faux est devenu évident, même s'il est rationalisé sous les auspices du raisonnable et du réalisme. Pour autant, ses dimensions apologétique et tragique demeurent latentes et n'ont pas été reçues comme telles à l'époque.

Les décennies de l'après-guerre ont vu s'amenuiser le crédit d'une discipline qui captait de moins en moins le prestige de la modernité et d'un discours d'actualité sur le monde, mieux endossé désormais par ces mêmes " concurrents ", économistes, sociologues, qui par ailleurs ne souffraient pas d'une image usée par les inculcations scolaires. II n'existe pas d'indicateurs qui permettraient d'objectiver aisément ce phénomène, mais tout porte à penser que le statut symbolique de la géographie a connu 
un lent déclin durant cette période, pour partie insensible, pour partie amorti au niveau universitaire par l'attractivité d'un cursus sans latin, par le caractère familier et peu spécialisé des études et enfin par une diversification thématique, en particulier en géographie humaine, qui a longtemps tenu lieu de modernisation. Cette décote externe correspond aussi à une dévaluation interne : si certains enseignants avaient un véritable rayonnement magistral, la littérature disciplinaire, privilégiant les manuels et les exercices pratiques, était perçue comme un compendium à usage alimentaire, loin d'une expérience de révélation sur le monde. Ceci posé, cette décote symbolique demeurait encore à l'état spectral dans le paysage des années 1960, moment où la demande de géographes croissait plus vite que l'offre, suscitant en interne des craintes de " pénurie " et consécutivement de " baisse de la qualité12"

L'essentiel du prestige scientifique était encore capté par une géomorphologie ${ }^{13}$ qu'E. de Martonne et ses successeurs avaient su imposer comme l'activité distinctive faisant la preuve de la valeur d'un géographe. Les géomorphologues occupaient une place déterminante, souvent majoritaire, dans les structures d'administration de la discipline. Ce sont eux également qui impulsaient des débats méthodologiques et éventuellement des controverses épistémologiques ${ }^{14}$. À l'appui de cette domination, les seuls laboratoires véritablement fonctionnels étaient de géographie physique et l'essentiel des crédits leur était échu. À tous les niveaux de la formation, le commentaire de cartes - visant au premier chef à « expliquer » le relief d'une « portion d'espace »-demeurait le plus impératif des exercices académiques, fourches caudines par lesquelles passait nécessairement la formation de géographes. La rénovation de la géographie humaine que l'on constate dans les années 1960 a été pour partie le fait de jeunes patrons (J. Beaujeu-Garnier, Philippe Pinchemel, Olivier Dollfus) qui avaient d'abord dû faire leurs preuves en géomorphologie pour être consacrés.

Davantage au fait des transformations structurelles en cours dans les sciences de la nature, les géographes physiciens ont souvent été à l'initiative de la diffusion de nouveaux standards épistémologiques : le développement de pratiques de laboratoire dès les années 1950, la mise en avant du travail collectif, I'accélération des échéances de publication, l'allégement des exercices canoniques, à commencer par la durée et le format de la thèse $d^{\prime}$ État $^{15}$. Dans la deuxième moitié des années 1960, ces perspectives sont devenues un enjeu catégoriel cristallisé autour d'attentes de réforme de la thèse

12 Rapport de J. Dresch aux Journées géographiques de Nanterre (1967) sur « les difficultés actuelles de l'enseignement et de la recherche en géographie », publié dans Intergéo, 8, 1967, ici 188-189.

13 Branche de la géographie étudiant les formes du relief et leur formation, et se spécialisant à l'époque dans les formes superficielles (modelé), dans un rapport complexe de forces et d'inclusions avec la géologie, discipline des facultés des sciences dont le capital symbolique ne s'est pas détérioré.

$\mathbf{1 4}$ Celles qui se développaient chez les spécialistes d' « humaine » apparaissaient davantage scolastiques, vite réinterprétées comme des conflits de personnes.

15 Claude Bataillon évoque dans Géographes. Génération 1930 (Bataillon, 2010) I'action en ce sens d'André Cholley, directeur de nombreuses thèses de géomorphologie, mais aussi la stratégie symétrique de Pierre George en géographie urbaine. 
d'État : I'Association des assistants et maîtres-assistants a soumis une motion en ce sens lors des Journées géographiques de Nanterre (février 1967) puis des chercheurs (contractuels) au CNRS prirent le relais en organisant un questionnaire interne dont la synthèse, sous forme de propositions, fut diffusée à l'automne 1967, en même temps que les résultats d'une enquête parallèle auprès des géographes ayant déjà soutenu leur thèse impulsée ${ }^{16}$ par le Comité national français de géographie (CNFG) ${ }^{17}$. La publication du tout dans le volume d'automne 1967 d'Intergéo manifeste assez significativement les rapports d'autorité régnant alors en géographie : un certain libéralisme autorise la mention et l'expression des élans réformistes des jeunes générations, adoubés sous forme d'une " proposition de résolution » devant le CNFG, mais la parole des géographes installés prédomine, ce qui s'exprime dans la matérialité même du dispositif. L'enquête auprès des " géographes-docteurs " est publiée en incipit du volume ${ }^{18}$ alors que le texte des chercheurs du CNRS validé, anonymisé, transformé en motion générale, est diffusé sous forme de feuille volante en recto verso. Les voix installées, citées en détail et souvent de façon nominative (sur quatre pages), ont le privilège de l'opinion individuelle. Si certaines des transformations revendiquées ${ }^{19}$ font l'objet d'un premier vote favorable, le compte rendu des « critiques » et " remarques" a des allures de retournement argumentatif en faveur du statu quo. De surcroît, aucune suite n'est formellement entérinée, laissant le sentiment d'un feu de paille, étouffé par I'hostilité des cadres intermédiaires de la profession (jeunes docteurs, maîtres de conférence, une partie des néoprofesseurs) ${ }^{20}$.

L'hétéronomie de décision des géographes sur les questions relevant de la politique universitaire (prérogative du ministère ou d'instances universitaires), l'absence de poids institutionnel du CNFG et le caractère fort peu représentatif de la consultation ramenaient de toute façon l'ensemble du processus à un simple geste consultatif, interpellant a minima la ligne de conduite des directeurs de thèse ${ }^{21}$. L'épisode atteste

\footnotetext{
16 Qualifiée de « référendum » au moment de sa conception en février 1967, elle n'a pas mobilisé (le taux de réponse fut de $20 \%$ ) et a été requalifiée en « enquête ».

17 Instance créée par E. de Martonne dans les années 1920 pour représenter la France dans les instances de l'Union géographique internationale (UGI). Dans les années 1960, seuls les docteurs d'État pouvaient en devenir membres. Ses moyens et prérogatives étaient faibles mais sa position de club sélectif et ses prétentions à légiférer sur le fonctionnement de la discipline en ont fait un symbole du pouvoir professoral à l'intérieur de la communauté.

18 Intergéo, 9, 1967. Ce volume est très mince (une vingtaine de pages). Pour une revue de littérature grise sans échéances rigides, I'hypothèse de contraintes de bouclage pour expliquer la dissymétrie de traitement n'est pas à exclure.

19 Elles systématisaient la publication d'articles en amont de la soutenance, limitaient la taille du volume soutenu tout en le vidant de sa dimension de "chef-d'œuvre ». D'autres revendications concernant la substitution de la thèse de $3^{e}$ cycle à la thèse complémentaire, la reconnaissance du travail collectif, l'implication du directeur de thèse, etc., figurent uniquement dans la proposition de résolution émanant des chercheurs au CNRS.

20 Le silence du compte rendu des Journées géographiques de Bordeaux (février 1968) dans Intergéo est à cet égard troublant.

$\mathbf{2 1}$ «Quoi qu'il en soit, avant toute législation nouvelle, une amélioration d'une situation généralement mauvaise dépend d'abord des directeurs de recherche. " : clausule de l'« Enquête sur la réforme de la thèse d'État ", Intergéo, 9, 1967, 259-262.
} 
néanmoins d'un fossé se creusant entre les " géographes-docteurs » (en particulier de fraîche date et sans chaire), souvent méfiants à l'égard d'un aggiornamento, signe pour eux d'« impatience » et de " facilité », et les générations montantes, porteuses d'une conception renouvelée de la recherche. Les patrons de la géographie française jouent un rôle discret, les uns (Jean Dresch, Pierre Monbeig) ayant encouragé l'initiative en favorisant sa diffusion, d'autres (Maurice Le Lannou, André Meynier, François Taillefer) en l'approuvant « sans réserves ", d'autres (Pierre Birot, Étienne Juillard, Louis Papy) en engageant un dialogue critique sur les modalités de la réforme.

On retrouve là le libéralisme souvent évoqué d'une génération d'hommes nés pour la plupart dans les années 1900-1910 et arrivés aux commandes de la discipline dans l'après-guerre, au moment de l'effacement graduel des élèves de Paul Vidal de la Blache, qui avaient fait le style universitaire de la géographie française depuis les années 1910. Parfois proches du Parti communiste français (PCF) sans être des marxistes doctrinaires (Pierre George, J. Dresch), la plupart étaient de sensibilité de " centre gauche ", comme en a fait état C. Bataillon dans diverses contributions ${ }^{22}$. Ils ont pour l'essentiel entretenu le legs de la tradition vidalienne dans un contexte d'expansion, accompagnant avec une prudence non dénuée d'un certain conservatisme épistémologique les développements tous azimuts de la géographie tant en matière d'objets, de thèmes que de méthodes (surtout en géographie physique pour cette dernière dimension). Les témoignages soulignent leur relative proximité avec leurs étudiants avancés, entre autres marques de relations sociales moins hiérarchisées et moins distantes que dans le reste des facultés de lettres. Dans les années 1960, ils ont par ailleurs encouragé les séjours à l'étranger non plus au motif du seul terrain de thèse mais pour formation, en particulier en Amérique du Nord, permettant à une poignée de doctorants de s'acculturer à la New Geography anglo-américaine. Ce mélange de bienveillance et d'encouragement implicite concourrait à un fonctionnement en petit monde relativement fluide et unitaire, jugé alternativement « familial » (P. Claval) ou « paternaliste » (C. Bataillon), en tout cas relativement consensuel. L'existence de réseaux divers (politiques, syndicaux, d'anciens élèves, de spécialités, etc.), contribuait néanmoins à différencier dans le détail un corps dont l'homogénéité sociale relative a longtemps nourri l'idéal unitaire.

Les années 1960 ont vu l'arrivée aux responsabilités des générations formées dans l'immédiat après-guerre, ayant connu ou immédiatement précédé le nouveau régime du cursus autonome de géographie, mis en œuvre à partir de $1944-1945^{23}$.

22 Déjà, dans « Table ronde imaginaire sur la géographie universitaire française 1930-1940 » (Bataillon, 1981), il faisait réfléchir plusieurs d'entre eux sur la spécificité d'engagements à gauche et au PCF des géographes de leur génération. $\mathbf{2 3}$ La création d'un cursus autonome de géographie (licence et agrégation) a été négociée par E. de Martonne auprès du régime de Vichy, aboutissant en 1943 après diverses tribulations. Accessible aux filières modernes, alliant signes de scientificité (via la géomorphologie et les pratiques de "terrain ») et valorisation du "concret » (une rengaine des géographes de l'après-guerre), il a abondamment contribué à accentuer les idiosyncrasies de la pratique disciplinaire. 
Cette génération de jeunes patron(ne)s peut notamment être caractérisée par une amorce notable de féminisation ${ }^{24}$, un recrutement social plus modeste, des sensibilités politiques plus différenciées (dont des personnalités franchement conservatrices), un attrait plus marqué pour les responsabilités politico-administratives. C'est parmi eux que s'est manifestée l'attitude la moins réceptive dans les débats de 1967 liés à la réforme de la thèse et une réticence à des changements qui auraient fait d'eux les derniers à en passer par les anciennes servitudes des humanités traditionnelles.

Ces brefs éléments de tableau suggèrent une trajectoire ambiguë, associant une expansion numérique et thématique typique des Trente Glorieuses au creusement de fortes différenciations internes, sur fond d'immobilisme doctrinal et d'usure latente du métier. Les effectifs d'enseignants du supérieur croissent considérablement, quasiment multipliés par cinq entre le milieu des années 1950 et 1967, avec un rapport cadres A/ cadres B qui passe de 2 à 0,5 entre 1956 et la fin des années 1960, créant les conditions de tensions de carrière dont l'analyse est classique pour donner un cadre à la crise universitaire de 1968. Pourtant, ici comme ailleurs, ce serait faire peu de cas de la dynamique de crise elle-même que de ramener les événements survenus en 1968 en géographie française à la manifestation d'une contradiction structurale.

\section{Mai-juillet 1968 en mosaïque et en demi-teinte}

Pour une large part, I'histoire des géographes en 1968 est une histoire locale, liée à des conditions disparates. Elle est parfois difficile à singulariser en raison d'une insertion encore étroite dans des facultés de lettres ou des collèges littéraires universitaires (CLU) de petite dimension ou très imbriqués. Parfois, à l'inverse, elle illustre assez bien les processus d'encellulement ${ }^{25}$ dont Antoine Prost a eu l'intuition dans son Histoire générale de l'enseignement et dont on commence à mieux documenter les ressemblances et les spécificités au regard du phénomène général d'occupation de locaux si fréquent en mai-juin $1968^{26}$. Le cas de l'institut de géographie de Paris, de loin le plus important et le plus autonome, est le mieux documenté, en tout cas du point de vue mémoriel. Plusieurs récits des événements provinciaux ont été donnés par des géographes en général chahutés à cette occasion : Alice Saunier-Seïté au CLU de Brest dans

24 On pourrait citer entre autres Jacqueline Beaujeu-Carnier (1917-1995), Renée Rochefort (1924-2012), Jacqueline Bonnamour (née en 1924), Alice Saunier-Seïté (1925-2003).

$\mathbf{2 5}$ « Le mouvement étudiant, ayant refusé de s'organiser, n'avait pas formulé de projet cohérent. Chaque cellule en grève, département de la Sorbonne, laboratoire, faculté de province, établissement secondaire, avait évolué à sa guise » (Prost, 2004, 346). Cet aspect d'entre-soi, omniprésent dans les monographies mais souvent peu valorisé dans les traitements globaux de l'épisode " 68 ", a ceci de significatif qu'il augure des innombrables initiatives à visée locale ou sectorielle que les auteurs de Mai-juin 68 (Damamme et al., 2008) ont appelé « subversions en pratique ».

26 Je renvoie pour comparaison à l'article de Patricia Vannier sur le Centre d'études sociologiques dans le même volume, et par ailleurs à l'article de Christophe Gaubert et Marie-Pierre Pouly sur l'institut d'anglais de la Sorbonne (Gaubert, Pouly, 2012). 
En première ligne. De la communale aux universités (Saunier-Seïté, 1982), Paul Claval à Besançon dans La géographie comme genre de vie. Un itinéraire intellectuel (Claval, 1996). Ils ont pour particularité, liée sans doute pour partie à la faiblesse des effectifs géographiques, de traiter des événements à l'échelle de l'établissement universitaire dans son entier ${ }^{27}$.

Si les crises sont locales, les confrontations ont entre elles un fort air de famille, pour autant que la documentation permette d'en juger. La période qui suit marque le glissement d'une perspective d'apparence unitaire qu'incarnent les « états généraux de la géographie » (juillet 1968) au clivage plus franc, quoique largement hétéronome, que manifestent les partitions dans les universités parisienne et lyonnaise au début des années 1970.

\section{Un entre-soi relatif}

Entre 1905 et 1930, la géographie française s'était dotée dans les principales villes universitaires d'une structure en « instituts de géographie » aux assises fort disparates, allant de la large autonomie de l'institution parisienne à la simple sectorisation dans des bâtiments communs, avec souvent une bibliothèque spécifique, des collections de cartes et quelques modestes installations ${ }^{28}$, sans que la géographie se différenciât physiquement comme on l'imagine ordinairement sous ce terme d'institut. En dehors de Paris, seuls les instituts de géographie de Grenoble (fondé par Raoul Blanchard en 1906 et largement subventionné par les milieux d'affaire locaux) et de Strasbourg, bénéficiaire des luttes d'influence avec l'Allemagne, ont disposé de locaux et de moyens significatifs, donnant à cette dénomination un caractère plus large que la revendication d'un entre-soi relatif. Après 1945, le développement de nouvelles universités (Nantes, Besançon, Limoges, etc.) et des CLU a ouvert un nouveau cycle de diffusion de la géographie dans des structures encore moins différenciées, où l'on parlait parfois simplement de "département » ou de " section ».

Dans ces conditions, il n'est pas étonnant que la contestation de mai-juin ait pris une tournure plus ou moins spécifique, largement indexée sur les échelles de pertinence de la contestation. Ainsi, à Toulouse, pourtant I'un des plus gros " instituts » de province par les effectifs, la dynamique fut largement transdisciplinaire autour du mouvement du 25 avril, impulsé par des philosophes et des économistes, dont

\footnotetext{
27 II y va aussi des fonctions de l'une (doyenne du CLU de Brest) et de l'autre, directeur de département et paritaire avant I'heure. P. Claval estime que sa posture à l'égard de la participation lui a permis de désamorcer toute humeur contestataire chez les étudiants géographes de Besançon, qu'il dépeint comme hostiles au mouvement. "Les étudiants de géographie refusèrent de se joindre au mouvement de contestation : la revendication essentielle était celle d'une représentation étudiante; ils étaient bien placés pour savoir que rien, dans les structures existantes, ne s'opposait à l'élection de représentants au niveau des sections et des départements » (Claval, 1996, 60). En l'occurrence, c'est à l'échelle de la faculté que se sont déroulés des conflits que P. Claval impute essentiellement à « la politisation du corps enseignant et la prise de pouvoir par les communistes » liées à des comportements politiques hégémoniques (Ibid., 61).

$\mathbf{2 8}$ Comme des tables de format inhabituel pour l'examen des cartes.
} 
I'une des préoccupations récurrentes était l'autoformation par l'entremise d'un projet d'université critique ${ }^{29}$. L'absence d'auto-organisation parmi les géographes locaux n'a pas empêché des revendications catégorielles d'émerger (mais " elles sont tombées à plat ») ni les affrontements directs avec certains " mandarins » hostiles au mouvement, en particulier Jean Demangeot ${ }^{30}$. Moment de mise en cause des rapports sociaux et politiques au sein de l'université, les occupations de mai-juin, à l'image de nombreuses crises ouvertes, sont un moment de libération du refoulé social, occasion d'exprimer, sinon de solder, des conflits jusque-là latents ou étouffés. Le rapport de force numérique substitué au rapport de force hiérarchique explique les formes plus ou moins accusées de chahut ayant frappé certains universitaires. Ce type de tensions, indépendantes du degré d'autonomie des géographes et réactivant parfois des contentieux plus anciens, intervient ou ressurgit dans bien d'autres lieux que Toulouse. II est attesté à Aix, mettant en cause les figures de Maurice Wolkowitsch ${ }^{31}$ et Pierre Gabert ${ }^{32}$, à Strasbourg, à Grenoble...

Dans les instituts de géographie les plus autonomes, matériellement distincts de leur faculté de rattachement, le processus d'encellulement est patent, même si la dynamique du mouvement ne s'y réduit pas : comme un nombre considérable d'autres lieux saisis par la dynamique révolutionnaire, leur fonctionnement durant les semaines d'occupation manifeste un double mouvement de connexion à la France en insurrection et de réforme locale. Ce sont en fait trois niveaux qui s'articulent dans les préoccupations des instituts en grève : une solidarité avec le mouvement national, qui se traduit par des votes de motions politiques et une participation plus ou moins coordonnée aux manifestations de masse; un élan réformateur visant le fonctionnement au quotidien des lieux et leur gouvernance; des propositions enfin d'aménagement des parcours nationaux de géographie, qui sont sans doute la part la

$\mathbf{2 9}$ « II y avait des revendications politiques générales et d'autres, universitaires. Les philosophes (Bensaïd, Cours-Salies) faisaient toujours la distinction. On allait tous à l'école, ces grandes AG dans les amphis. On retrouvait le statut d'étudiant qui avait tout à apprendre » (entretien, géographe assistant à Toulouse en 1968). Les figures les plus connues sont Daniel Bensaïd et Alain Alcouffe, ce dernier ayant consacré des éléments de blog à cette thématique (http://alain.alcouffe.free.fr). Voir aussi C. Fauré (1988) et B. Bennassar (2000).

30 Élève d'Emmanuel de Martonne, spécialiste de géomorphologie tropicale, Jean Demangeot (1916-2009) a soutenu une thèse relativement sur le tard (1965) avant d'échanger un poste de maître de conférences à Reims avec celui de Roger Brunet à Toulouse. En conflit avec les assistants locaux dès l'année 1966-1967, en particulier sur ses exigences en matière de commentaire de cartes, il a très mal vécu les événements de 1968 et profité de la première opportunité pour rejoindre Nanterre, université où nombre d'enseignants de géographie étaient assez conservateurs et partageaient son horreur de 68.

31 Spécialiste de géographie des transports et secondairement de la Tunisie, M. Wolkowitsch (né en 1920) avait soutenu sa thèse de doctorat, L'économie régionale des transports dans le Centre et le Centre-Ouest de la France, en 1960. II était professeur à Aix-en-Provence depuis le milieu des années 1960 et incarnait une géographie thématique sous cadre régional. 32 Géomorphologue, assistant de Pierre Birot à Paris pendant cinq ans, auteur en 1962 d'une thèse sur Les plaines occidentales du Pô et leurs piedmonts, Pierre Gabert (né en 1927) a été très rapidement nommé professeur à l'université d'Aix-en-Provence et a été précocement impliqué dans les instances nationales de la géographie, notamment au jury de l'agrégation. 
moins révolutionnaire de l'activité des instituts occupés. Comme ailleurs, on observe une fraternisation entre diverses catégories d'usagers (enseignants, le plus souvent de " rang $B$ ", étudiants, personnel administratif et de maintenance) réalisant à travers l'investissement et l'entretien des lieux, ici particulièrement organisé (comme le révèlent différents témoignages), une expérience concrète d'autogestion.

Au sein du mouvement, l'institut de géographie de Paris est sans doute celui qui a incarné le plus complètement une expérience de " commune géographique " à l'œuvre durant la période. "Navire amiral » de la discipline, regroupant plus du cinquième des effectifs étudiants et du personnel du supérieur en France ${ }^{33}$, situé en surplomb du quartier latin, à la croisée des rues Saint-Jacques et Gay-Lussac, il fut occupé à partir du lendemain de la nuit des barricades (10 mai), qui ici comme ailleurs créa un effet de sidération et dramatisa l'affrontement avec le pouvoir gaulliste et la " société capitaliste ». Du fait de sa position péricentrale, il ne fut pourtant jamais investi par les forces de l'ordre ou attaqué par les groupes d'extrême droite, mais fut l'objet d'une attention scrupuleuse de ses occupants, qui en firent durant un mois et demi un lieu de vie et de débats (diurnes) permanents. Son fonctionnement au quotidien avait un air de famille marqué avec ce qui a pu être décrit pour d'autres centres de recherche ${ }^{34}$ ou d'autres établissements spécialisés, comme l'institut d'anglais de la Sorbonne (Gaubert et Pouly, 2012). A contrario de la peur des "nouveaux barbares» telle qu'elle transparaît dans les témoignages d'universitaires traditionnels (exemples dans Callu, 2010), la préservation des biens et des bibliothèques fut une hantise des occupants et traduit un souci patrimonial qui mériterait une analyse plus approfondie. Outre des assemblées générales quasi quotidiennes, le personnel en grève a produit de nombreux groupes et commissions : service d'ordre, comité de grève et d'action, commissions « pédagogie ", " structures de l'université ", " moyens d'action ", " université et société $" . . .{ }^{35}$ Les motions des AG révèlent un va-et-vient constant entre les trois niveaux de préoccupation déjà mentionnés, avec une énonciation comme acteur collectif se positionnant sans relâche sur une scène nationale, à la fois politique et universitaire, et multipliant les adresses au " gouvernement ", aux "forces de gauche " et à d'autres acteurs de premier plan.

Dans ce mouvement, certains étudiants ont joué un rôle de premier plan, tant du point de vue de la réflexion politique que de la capacité à se poser en interlocuteur des enseignants, que ces derniers soient mobilisés, attentistes ou hostiles. En particulier,

33 Selon diverses statistiques avancées à l'époque, il y avait environ 10000 étudiants en géographie en France, et plus de 2000 dans le seul institut parisien. Ce ratio du cinquième, à peu près équivalent pour le personnel universitaire (Robic, 1989) n'est pas considérable, si on le compare à la situation de sciences plus récemment institutionnalisées (sociologie, économie) où la place du pôle parisien était nettement plus marquée. Il signe a contrario le poids relativement important de la province dans une discipline décentralisée plus tôt que d'autres.

34 Voir l'article de P. Vannier sur le CES dans le même volume.

35 Cet inventaire est sans doute incomplet, compte tenu des lacunes chronologiques dans les comptes rendus d'assemblées générales disponibles, principale source sur laquelle je m’appuie pour analyser les événements en géographie parisienne. 
un groupe de jeunes femmes a marqué les esprits, dont les figures marquantes furent Hélène Lamicq et Claire Biétry, régulièrement citées par les différents protagonistes de l'époque. Une dimension charismatique semble avoir fortement joué parmi les étudiants mobilisés, fût-ce de manière provisoire. Les aspects spécifiques de cette mobilisation au sein du mouvement demeurent flous et demandent de nouvelles enquêtes.

L'attitude du personnel dirigeant de l'institut a été fort variable. Son directeur, Jean Dresch, qui détenait à l'époque ${ }^{36}$ de nombreux leviers de pouvoir disciplinaire au niveau national, s'est associé au mouvement durant toute sa durée, conservant intact tel un équilibriste la plupart de ses prérogatives d'administrateurs du centre, et conforté par des élections à la fin juin 1968 : à l'occasion d'un vote par collèges les 20 et 21 juin, suivi de l'élection d'un bureau, il est confortablement reconduit dans ses fonctions de directeur. À la différence d'autres géographes engagés au parti communiste, J. Dresch n'a manifesté aucun signe d'hostilité aux grévistes, auxquels il a fourni l'appui de sa position antérieure et de ses réseaux à travers la France ${ }^{37}$. Plus ambiguë fut l'attitude de professeurs tels J. Beaujeu-Garnier, P. Pinchemel ou Christian Sautter, qui assistèrent à nombre d'assemblées générales ou de débats tout en maintenant la dynamique protestataire à distance au nom de l'idée qu'ils se faisaient de leur statut social ou du fait de leurs convictions politiques, sans parler de la posture goguenarde assez répandue parmi les universitaires français ${ }^{38}$. En revanche, nombre de "maîtres » demeurèrent à l'écart des événements, comme en atteste le comportement de Pierre George, figure intellectuelle majeure de la géographie française de l'époque, demeuré très en retrait durant l'essentiel de la crise. Michel Rochefort, ancien élève du précédent, élu de fraîche date dans une chaire de géographie économique, fut le seul " rang $A$ » à s'inscrire dans le mouvement ${ }^{39}$. Il a décrit son rôle de go-between entre la jeunesse occupant l'institut et les " mandarins », organisés par et autour de J. Beaujeu-Garnier pour surveiller la situation et maintenir en dehors des lieux occupés un môle de pouvoir traditionnel (dans l'appartement qu'elle habitait à proximité de la rue Saint-Jacques). Pour autant, les relations directes dans les assemblées générales semblent être demeurées feutrées et assez peu conflictuelles, et même marquées par un reste de déférence, dont atteste

36 Cf. supra note 7.

37 On peut faire I'hypothèse que les origines bourgeoises de J. Dresch expliquent son attitude différente de celle d'autres communistes, d'origine populaire et arrivés « au mérite », qui ont davantage partagé l'hostilité de la direction de leur parti à l'égard d'un mouvement jugé étranger à sa tradition et à ses positions. À cela s'ajoutait le rejet ambiant du « stalinisme » et de toute forme d'autoritarisme, y compris ouvriériste.

38 La dérision à l'égard du mouvement est attestée chez P. Pinchemel et C. Sautter. Le témoignage de I'historien B. Benassar sur son expérience toulousaine (Benassar, 2000) est particulièrement emblématique de cette réaction « dispositionnelle » dont on trouvait déjà des signes dans les premiers essais d'égohistoire (Nora, 1987).

39 Son témoignage, recueilli par Nicole Mathieu et Stéphane Cartier (Rochefort, 2002), est un précieux document sur l'ambiance qui régnait à l'époque, à la croisée des « patrons » et du mouvement. Sur son parcours et l'élucidation de sa position de géographe de gauche, élève de P. George, rénovateur de la géographie française dans les années 1960, voir Bataillon, 2006. 
une réunion sur les perspectives de professionnalisation où furent officiellement invités et attentivement écoutés ${ }^{40}$ les patrons jusque-là renvoyés à la condition de spectateurs et de participants occasionnels.

En définitive, si la disposition séparative des instituts de géographie a favorisé un certain entre-soi parmi les géographes et une intense réflexion "localiste ", ce ne fut sans doute pas au détriment d'un positionnement politique national ${ }^{41}$, sensible aux soubresauts de la crise, la contestation géographique mêlant ses voix et faisant corps avec le mouvement d'ensemble. L'hybridation semble avoir été la règle, articulant une perspective révolutionnaire sur le plan national et des aspects nettement plus réformistes, sinon conformistes, d'un point de vue local ou disciplinaire ${ }^{42}$. Ces derniers constituent le gros des archives - par contraste avec une parole politique qui a laissé peu de traces autres que mémorielles - et permettent de cerner les contours du répertoire revendicatif dans ses dimensions fonctionnelle et académique.

\section{Un réformisme conformiste?}

Les revendications manifestes de la production contestataire font émerger assez banalement deux perspectives fortes. L'une est adossée à un rejet quasi unanime de la réforme Fouchet ${ }^{43}$ - qui venait seulement d'entrer en application à la rentrée 1967 d'où ressortaient une spécialisation disciplinaire jugée trop précoce et un sentiment d'ajustement au marché de l'emploi lu à l'époque en termes de « soumission aux impératifs du capitalisme ». L'autre est la demande tous azimuts de paritarisme, au premier chef appliquée au fonctionnement des instituts et sections de géographie, éventuellement étendue à la gestion des examens ou des centres de recherche. De façon assez significative, l'élan réformateur dépassait I'horizon de proximité qu'on lui imaginerait en première analyse : les étudiants parisiens ont participé à l'élaboration de statuts d'un "centre de recherches géographiques de Paris-Sorbonne ", a priori extérieur à leurs préoccupations directes; ceux du CLU de Brest ont élaboré un projet de réforme de l'université française, etc. Ces initiatives sont le signe d'une curiosité et de perspectives dépassant largement l'horizon des pratiques connues et ordinaires, traits que l'on retrouve aussi dans des documents plus « politiques » produits durant ces semaines d'effervescence.

Les réformes des « structures» (de gouvernance, dirait-on aujourd'hui) sont à la fois très mobilisatrices et celles qui ont un rendement relativement immédiat : dès la

40 Je n'ai pu pour l'instant ni la dater précisément ni en retrouver un verbatim.

$\mathbf{4 1}$ En guise d'exemple, je donnerai une motion de soutien à D. Cohn-Bendit présentée le 23 mai et votée le lendemain, suscitant toutefois légèrement plus d'opposition que les motions ordinaires soumises en AG.

42 Traits au demeurant déjà notés par N. Mathieu (dans Rochefort, 2002).

43 Déjà, un « dossier » de février 1967 de I'Union des géographes de la faculté des lettres (UGFL) de Paris (affiliée à I'UNEF), intitulé " Pour une meilleure formation du géographe », avait été diffusé pour dénoncer l'application annoncée de la réforme et réfléchir au statut social et au devenir des étudiants de géographie. 
mi-juin, les instituts de géographie de Paris et Grenoble ont ainsi disposé de "s statuts » paritaires ouvrant sur des élections et un fonctionnement « co-gestionnaire » qui allait se prolonger au-delà des événements proprement dits. Dans les départements moins autonomes, tel celui de Poitiers, il semble que les géographes se sont impliqués dans un paritarisme à l'échelle de la faculté tout entière ${ }^{44}$. Dans les lieux où la participation des étudiants avait déjà été encouragée, ce précédent a pu avoir un effet démobilisateur (cas de Besançon, cf. supra note 27) ou donner lieu à des malentendus alimentant une conflictualité importante (cas de Strasbourg).

Au-delà de la nécessité d'une abolition d'un « plan Fouchet » honni, c'est la question de l'enseignement de la géographie, à tous les niveaux, qui a suscité les plus forts élans réformateurs. Ainsi, la commission « pédagogie » de l'institut de géographie de Paris a-t-elle produit pas moins de quatre documents différents, concernant d'une part "l'enseignement de l'histoire et de la géographie dans le secondaire » et d'autre part les études supérieures dans une large gamme de perspectives - l'une d'entre elles, clivante au sein même du mouvement, concernant le cursus de géographie, au sein duquel l'équilibre entre pluridisciplinarité et spécialisation faisait débat. À l'unisson de leurs pairs, les géographes aspiraient à un allongement généralisé des formations (à quatre voire cinq ans), à une réduction de l'enseignement magistral, corrélat de la mise en avant du travail de groupe, et à une plus grande latitude dans la composition des parcours. La question de I'« autonomie » était fortement débattue, mais sous des interprétations fluctuantes et relativement ambiguës ${ }^{45}$. En revanche, les grands topoï de la pratique disciplinaire - pratiques de terrain et notamment d'excursion, rapport à la carte (produite ou commentée), grands partages thématiques de la discipline, etc. - ne faisaient pas l'objet d'une quelconque distanciation et étaient au contraire mis en avant comme allant de soi. À peine voit-on apparaître un appel pressant au développement de l'enseignement des statistiques. Autrement dit, si les géographes contestataires étaient au diapason d'un fonctionnement universitaire en recomposition rapide et manifestaient un intérêt significatif pour les questions pédagogiques, leur perspective critique cédait largement le pas au conformisme sur le chapitre du contenu disciplinaire.

Néanmoins, les témoignages, disponibles ou que j'ai recueillis, font état d'interrogations récurrentes sur l'identité et la place de la géographie. II semblerait que ces questions lancinantes ont été largement suscitées par le flou planant sur l'organisation et le statut des professionnalisations alternatives aux carrières de l'enseignement, en particulier dans un contexte de concurrence et de précarité sur le marché

$44 \mathrm{~J}$. Dresch avait ainsi archivé un courrier du 25 juin de Jean Cabot (Poitiers) lui transmettant les statuts élaborés dans sa faculté. J. Cabot sera ensuite membre du «noyau cooptant » de Vincennes, éphémère président de cette université, avant d'en partir précocement (voir Soulié, 2012).

45 Comme en atteste un compte rendu d'une commission paritaire de Nanterre, en date du 22 mai 1968, très hésitant sur la sémantique du mot « autonomie». 
de l'aménagement : ces problèmes ont occupé une place non négligeable dans des débats d'AG (hélas très rarement retranscrits) de mai-juin 68. Sous réserve d'examens complémentaires, cette indexation d'un débat potentiellement épistémologique sur des perspectives professionnelles ou d'utilité sociale éclaire une réponse relativement spontanée (i.e. peu réfléchie) qui fut souvent répétée et fonctionnait par renchérissement d'un credo bien connu : le géographe est « homme de synthèse » des " situations localisées ", la géographie, " discipline-carrefour », " science du concret "... Ces formulations, inscrites dans la tradition des décennies précédentes ${ }^{46}$, sont peut-être éclairées par le contexte d'incertitude d'une crise politique au tempo saccadé et faisant largement droit à l'improvisation et au « bricolage » (Gobille, 2008b). Elle fait contraste avec les débats proprement épistémologiques de la décennie ultérieure, qui semblent avoir été de l'ordre de l'inconcevable en 1968.

Au demeurant, dans les instituts de géographie où s'était développée une culture de la « géographie appliquée » (Rennes, Strasbourg) cette question était plus sensible qu'ailleurs. Déjà, en 1967, le rapport (parisien) de I'UGFL " Pour une meilleure formation du géographe » critiquait une gestion des contrats totalement à la main des professeurs, à court terme, et reposant sur l'aliénation du travail et des perspectives des étudiants recrutés, faute d'une labellisation par le diplôme et d'une « rationalisation » des critères d'embauche. À Strasbourg également, des tensions étaient apparues en 1966-1967 (Rebeyrol, 1968). Dans la période de contestation qui s'ouvre en maijuin 1968, il semble que le malaise engendré par ces pratiques de placement se soit généralisé et explique notamment les formes durables de chahut ayant frappé Jean Tricart à Strasbourg ${ }^{47}$, vécues par l'intéressé comme incompréhensibles au regard de son engagement (communiste) et de ses pratiques (dévouées et démocratiques à ses yeux, "paternalistes " pour les étudiants)... Cette situation d'incompréhension réciproque manifeste la dimension conflictuelle de la période : les relations sociales enseignants/enseignés, professeurs/assistants, etc., sont à la fois plus intenses quand elles ont lieu d'être durant ces mois de prise de parole (de Certeau, 1994), à distance du paternalisme un peu familial de la séquence qui précède, et plus directes. Mais elles augurent de potentiels ou déjà bien réels conflits, engendrant des ressentiments et des perplexités. Dans son histoire de la discipline parue en 1969, André Meynier conclut son ultime chapitre intitulé « Le temps des craquements » par une brève évocation du

\footnotetext{
46 Réponse aux défis de l'aménagement (identité scientifique, place dans le « concert » des sciences) qui avait déjà été celle, pour le coup très élaborée, d’un P. George ou d'un J. Labasse dans les années 1960. Voir le chapitre « Défense de la doxa et incommensurabilité paradigmatique » de mon livre De plain pied dans le monde... (Orain, 2009a, 179-219).

47 Jean Tricart (1920-2003), géomorphologue hétérodoxe et novateur (il a introduit une approche climatique de l'évolution des reliefs), mal vu par ses collègues pour son engagement communiste qu'il entendait appliquer à l'exercice même de la science naturelle. II a largement contribué à une réflexion méthodologique sur la géographie physique augurant du tournant systémique et " global » qu'elle a pris dans les années 1970. À l'origine d'un projet (avorté) de diplôme d'expert-géographe en 1958, il était, avec M. Phlipponneau à Rennes, I'un des plus actifs négociateurs de contrats et « plaçait » des étudiants à tour de bras.
} 
climat de l'époque, dont il retient les antagonismes nombreux et « un certain parfum d'intolérance " (Meynier, 1969, 216). Contrairement à un motif trop simple, I'humeur polémique n'est pas entièrement cristallisée au sortir des occupations de mai-juin 1968. Dans un premier temps, l'urgence est plutôt à la (ré)union des géographes, dont témoigne l'organisation d'« États généraux de la géographie " au début de l'été.

\section{Des " États généraux de la géographie "}

Jean Dresch, fort de ses réseaux et de ses nombreuses responsabilités au sein de la communauté des géographes, mais aussi de sa position nodale durant le printemps, a semble-t-il été à l'origine d'une initiative pour réunir des représentants de toutes les " sections » de géographie universitaire de France, qui s'est tenue à Tours du 10 au 12 juillet 1968. Construits selon l'esprit paritaire du temps, avec des règles assez strictes (deux enseignants et deux étudiants par établissement et par jour) ${ }^{48}$, ces " États généraux » n'ont pas été les seuls du genre et correspondent plus ou moins à un modèle de forum qui a connu alors un fort engouement, dans un continuum entre un pôle " université d'été » (centré sur un objet extérieur) et un pôle " assises » (centré sur un groupe réfléchissant à son avenir). L'aspect très institutionnel de cette opération contraste avec d'autres développements estivaux à la dynamique plus spontanée, telles ces universités d'été ou initiatives de terrain auxquelles ont participé avec enthousiasme de nombreux géographes, entre soif d'enquête, travail d'équipe et aspiration à se mettre au service des " masses ".

L'événement est très bien documenté : sa retranscription a fait l'objet d'un numéro d'Intergéo (Collectif, 1968), très sténographique dans son rendu et nécessairement policée (ce qui en constitue la limite principale pour saisir l'humeur du moment) ; il a donné lieu à un article dans Le Monde (Rebeyrol, 1968) qui en propose une vision extérieure dynamique. Grâce à quoi on peut notamment réfléchir à ses caractéristiques géographiques et sociales : des centres importants sont absents (Nancy, Rennes, Strasbourg ${ }^{49}$ ), les listes de participants inscrits ${ }^{50}$ dessinent une géographie des rapports de force noués au printemps, avec des délégations réduites à un universitaire (P. Claval représente Besançon, J. Bastié Nanterre $\left.{ }^{51}\right)$, d'autres qui respectent la prééminence des patrons (Bordeaux, Lille, Paris, Poitiers), d'autres où assistants, maîtres-assistants et étudiants jouent un rôle central (Aix-en-Provence, Caen, Grenoble, Lyon, Nantes, Pau,

48 La rigidité des règles de représentation a été déplorée par la commission paritaire de l'institut de Paris à la fin juin.

49 Faut-il y voir le reflet de tensions particulièrement vives (Rennes, Strasbourg) ou d'une prééminence absolue de mandarins de droite très hostiles (Nancy)?

$\mathbf{5 0}$ C'est une nuance importante : sauf à retrouver les noms consignés de manière non anonyme dans le compte rendu des débats ou de recueillir des souvenirs, la présence effective n'est pas garantie par ces listes.

$\mathbf{5 1}$ L'un et l'autre très hostiles au mouvement et pour lesquels la période a joué un rôle de bascule décisif. Jean Bastié (né en 1919), d'abord instituteur puis professeur de centre d'apprentissage, proche du PCF jusqu'en 1968, avait échoué maintes fois à l'agrégation et fut longtemps assistant dans I'ombre de P. George. II a soutenu en 1964 une thèse sur La croissance de la banlieue parisienne. Son rôle après 1968 est évoqué infra. 
Toulouse), d'autres dont la participation s'est étiolée avant terme (Brest, Limoges, Montpellier, Rouen). Ces participations différentielles ont sans doute des significations diverses et pour partie contingentes : les vacances ont commencé, dont toute l'historiographie souligne la fonction de pause après la crise.

La première journée (8 juillet) installe des commissions " pédagogie ", " structures » et "débouchés, recherche, enseignement ». Les journées des 9 et 10 juillet sont consacrées à des débats à partir des travaux de celles-ci. Durant les deux premiers jours, en particulier le premier, se lit une dominance des universitaires installés, avec une forte prépondérance de paroles individuelles qui disent ce qui est ${ }^{52}$. La situation évolue le deuxième et surtout le troisième jour : les " patrons " sont partis (J. BeaujeuGarnier, P. Claval53) ou sont moins présents; il en reste une poignée, certains en affinité avec le mouvement (Roger Brunet), d'autres dans un dialogue vigilant (Pierre Brunet, Pierre Flatrès, Guy Lasserre). Au milieu de débats intenses mais relativement bonhommes, Jean Bastié s'isole parfois dans une posture de défenseur de la tradition ${ }^{54}$ dont les motions sont rejetées. Certains assistants s'illustrent par une posture parfois incisive (Marceau Rochette ${ }^{55}$, Maurice Allefresde ${ }^{56}$ ). L'après-midi du mercredi 10 juillet, sous la présidence de J. Dresch, est consacré au vote de motions au caractère souvent plus radical que celles présentées précédemment ${ }^{57}$, séquence durant laquelle l'assemblée générale est donnée comme un acteur collectif quasi organique : il n’y a plus d'individus, uniquement une voix souveraine et unanime. Les rangs sont raréfiés, il n'y a plus de décompte précis des voix, la langue devient nettement moins « techno » et prend des consonances soixante-huitardes ${ }^{58}$.

Dans ses thèmes et ses accents, cette assemblée paritaire a beaucoup de traits communs avec les thèmes réformistes analysés précédemment. Sur le brûlant problème

$\mathbf{5 2}$ Le compte rendu n'est pas homogène pour des raisons difficiles à démêler : les participants sont parfois désignés par leur nom et d'autres fois ramenés à leur origine géographique.

53 II n'avait pas encore soutenu de thèse (ce sera chose faite en 1971) et n'avait pas statut de professeur ou de maître de conférences (ancien régime), mais son autorité d'auteur déjà connu et de directeur du département de Besançon se ressent jusque dans la forme de ses prises de parole.

54 Par exemple, lors d’un débat sur les « domaines réservés », il « se déclar[e] effaré de voir avec quel sectarisme on considère les institutions anciennes comme mauvaises » (collectif, 1968, 191).

55 René Marceau Rochette (1933-2012) a fait toute sa carrière à Grenoble. Longtemps proche des troskystes, il a consacré ses travaux au Sahel et aux conséquences de la désertification.

56 Maurice Allefresde (1926-2002), militant anarchiste, a été l'un des premiers promoteurs en géographie d'une approche du développement rural par la base, notamment appuyé sur le motif de " petites cellules rurales » et la formation d'animateurs locaux, et ce dès le milieu des années 1970. Sa thèse porte sur les dynamiques de la vie rurale dans les marges de l'œkoumène en Scandinavie.

57 Elles ont pour origine un second rapport de la commission " pédagogie » (débarrassé de la tutelle des patrons?) où I'on voit apparaître le thème de l'implicite et de l'explicite des " présupposés idéologiques », texte qui sera adopté point par point durant ce mercredi après-midi à l'ambiance plus conforme à une certaine imagerie de l'époque.

58 Exemple : «Constatant que le savoir n'est pas un donné définitif et que sa transmission nécessite un examen critique constant, la commission demande une mise en perspective scientifique et idéologique du contenu de l'enseignement » (210). 
des débouchés hors enseignement, elle botte en touche, s'en remettant à une enquête et à un " grand colloque » (qui l'un et l'autre n'auront pas eu lieu), tout en remettant en cause l'autonomie des IUT et des formations techniques, donnés comme une forme aliénée de formation (mais aussi comme concurrents à une géographie formatrice de « cadres moyens » de l'aménagement?). Le principe d'une évaluation des enseignants par les étudiants donne lieu à un vote favorable. La critique des « présupposés idéologiques » du cours magistral s'accompagne d'une promotion des « travaux de terrain » donnés comme consubstantiels à la géographie (et donc obligatoires) et d'une autogestion en groupes de travail étudiants.

Si cet épisode est intéressant pour se faire une idée d'une part des possibilités de dialogue dans la géographie française au sortir de deux mois de grèves et de contestation et d'autre part des modalités redevenues hiérarchiques de la prise de parole dans une arène de l'immédiat après-68, force est de constater qu'il a eu une répercussion minime : du fait de l'hétéronomie de décision des géographes sur les questions de politique universitaire, ses motions sont restées largement lettre morte; des participants enregistrés n'en ont gardé aucun souvenir ; l'agenda de suivi n'a pas été respecté. Compte tenu de l'amnésie qui l'a frappé, à l'image de nombreuses actions prolongeant le mouvement dans l'été 68 , il serait tentant de le considérer comme une initiative sans lendemain. II témoigne toutefois de ce que l'impression de dégâts irrémédiables (Claval, 199859) n'était pas encore cristallisée et que la fracture se conçoit mieux comme un processus étal, se prolongeant sur plusieurs années. L'examen d'autres moments collectifs, comme les Journées géographiques de Rouen (1969), par moments assez houleuses, permettrait sans doute d'affiner la compréhension du processus de fracture qui s'est approfondi graduellement entre 1968 et 1972.

\section{De la fracture politique à la crise épistémologique (1968-1976)}

\section{L'amorce d'un processus de fracture et de polarisation}

Au-delà des événements proprement dits, l'impression d'une période de fractures multiples fait consensus dans la mémoire collective et se laisse également deviner dans une partie de la littérature disciplinaire. De façon assez caractéristique, plusieurs livres publiés entre 1969 et 1971, mais dont l'écriture avait commencé antérieurement à la crise, se firent l'écho de tensions émergentes. C'est le cas de l'Histoire de la pensée géographique en France d'André Meynier (1969) dont l'épilogue oscille entre un panégyrique de la discipline et l'évocation des « conflits » et « excommunications » régnant en son sein. C'est la fin du « Que sais-je? » que Pierre George consacre aux Méthodes

59 Voir notamment les développements des pages 330-331, qui présentent un tableau noir et désenchanté de la séquence, marquée par une pulvérisation de la communauté et une " division des esprits ». 
de la géographie (1970), qui se conclut de façon assez imprévisible sur l'annonce d'un " procès" (au sens judiciaire) et d'une " crise » de tout ou partie de la géographie - lequel motif de la " crise " allait être abondamment repris dans les années suivantes. C'est encore le livre assez étrange de J. Beaujeu-Garnier, La géographie : méthodes et perspectives (1970), dont I'arrière-plan implicite est une contestation à laquelle il faut opposer de l'« incontestable ", fût-ce au prix d'une improbable "synthèse entre la tradition et la nouveauté » (avant-propos) ${ }^{60}$. Pourtant, cette crise annoncée apparaîtrait ténue si elle n'avait trouvé des modes d'objectivation politico-institutionnels spectaculaires.

La répercussion directe la plus éloquente fut d'ordre politico-syndical. La (re)fondation d'une Fédération des syndicats autonomes de l'enseignement supérieur (FSAER) en réaction à Mai 68 (Déplaude, 2009) annonça l'émergence d'un bloc d'universitaires hostiles à l'évolution " gauchiste » du Snesup et du SGEN et décidés à lutter contre les changements revendiqués dans les amphis de mai-juin. En géographie et même au-delà, J. Bastié a joué un rôle semble-t-il très important dans la constitution d'un front puissant ${ }^{61}$, immédiatement majoritaire chez les « cadres $A$ » de la discipline. De nombreux anciens adhérents du Snesup passèrent alors d'un « centre-gauche » assez flou à un ancrage définitif à droite. Le cas d'Alice Saunier-Seité, future secrétaire d'État puis ministre des années Giscard, apparaît comme un exemple emblématique de ces basculements, qui furent légion. Claude Bataillon a analysé un profil typique de géographe d'origine modeste et à la carrière laborieuse et tardive qui a basculé en 1968 chez les autonomes, choqué par les critiques visant les « mandarins » et la remise en cause abrupte du système de valeurs sur lequel s'était appuyée son ascension sociale ${ }^{62}$. Au-delà, le front du rejet de 68 a des caractéristiques intergénérationnelles et a enrôlé des géographes plus jeunes et tôt marqués à droite, comme $P$. Claval ou Jean-Robert Pitte $^{63}$. A contrario, certains patrons " modérés » au rayonnement intellectuel plus

\footnotetext{
60 J'ai analysé ces ouvrages dans De plain-pied dans le monde (Orain, 2009a, chap. 4 et 5) et leur participation à un « malaise » de la géographie française qui prélude à la crise épistémologique des années 1970.

61 On dispose d'un écho de son activisme dans les souvenirs de Jean Demangeot: " Avec les événements de Mai 68, mon collègue géographe Jean Bastié, engagé à droite, mais il avait été auparavant d'extrême gauche, me poussait à choisir mon camp et à le rejoindre dans son syndicat de droite, sinon me disait-il "tu seras mangé" » (Daudel, 2008, 20). 62 C. Bataillon, "Le système institutionnel des géographes en France et 1968 ", texte inédit. II cite entre autres les cas de Georges Viers (1910-1998), André Journaux (1915-2006), Jean Bastié (né en 1919), Guy Lasserre (1920-2001), Michel Laferrère (né en 1924) et Pierre Gabert (né en 1927). Le cas du premier (demeuré membre du PCF) est sans doute à distinguer, même si des géographes de bords politiques en principe opposés se sont rejoints en 1968 dans une commune détestation du mouvement contestataire. II y adjoint Alain Huetz de Lemps (né en 1924) dont le background (famille d'extrême droite, père condamné à la Libération) est très différent et relèverait selon moi d'un autre profil. Cette question de la part relative de l'origine sociale, de la socialisation politique initiale et du parcours académique dans la réaction aux événements de 68 demande à être approfondie.

63 Né en 1949, il entame ses études à la Sorbonne à l'automne 1966. Deux ans plus tard, il devient le leader des étudiants opposés au mouvement et dirige avec A. Louchet une liste « Défense de l'université » qui siège dans le second conseil paritaire, installé en mars 1969. II a raconté son Mai 68 à l'envers dans le livre de S. Allemand, Comment je suis devenu géographe? (Allemand, 2007, 158).
} 
assuré (Philippe Pinchemel, François Taillefer) et dont la position sociale relevait d'une autre forme de trajectoire, ont pu regarder le mouvement contestataire avec une certaine sympathie et se tenir plus tard à distance de ce front des " anti ». Ce dernier, devenu majoritaire dans les comités nationaux, exprima sa vision tout au long des années 1970 dans les choix de subvention aux équipes de recherche et les décisions de classement et d'avancement des carrières.

Parmi les répercussions directes figurent aussi certaines conséquences des réformes $d^{\prime}$ Edgar Faure. L'effort de restructuration en UER et universités eut pour revers d'importantes contractions budgétaires touchant les structures anciennes, en particulier celles qui étaient devenues des communes autonomes et des foyers de contestation : l'institut de géographie de Paris, au bord de l'asphyxie durant les années suivantes ${ }^{64}$, semble ainsi avoir été mis à la diète. Endiguement d'un côté, exutoire de l'autre, on pourrait lire les incitations au mouvement vers I'université de Vincennes créée à la même époque comme une manière de confiner les éléments les plus voyants du gauchisme universitaire dans un réduit mal doté65. Au demeurant, pour ce qui concerne la seule géographie, hormis les figures de Raymond Guglielmo et d'Yves Lacoste, I'université " expérimentale " n'a pas été un point de ralliement " gauchiste » ${ }^{66}$, attirant même des géographes plutôt classés à droite. Encore s'agit-il d'un mouvement quantitativement secondaire en regard du processus de partition qui s'opéra en Sorbonne après deux années d'hésitations (été 1970). En ce qui concerne la géographie, celui-ci s'effectua de façon peu originale entre un Paris IV scientifiquement et politiquement conservateur ${ }^{67}$, un Paris VII plutôt marqué à gauche, localement porté sur la réflexion didactique, les pays en voie de développement, la géographie physique, le gros des effectifs ralliant Paris I et une position médiane, fédérant droite moderniste (J. BeaujeuGarnier, Jacqueline Bonnamour), rénovateurs démocrates chrétiens (P. Pinchemel, C. Sautter) et divers positionnements de gauche. Au demeurant, les trois universités issues de la réforme Faure ont continué à cohabiter dans les locaux de l'institut de géographie de Paris ${ }^{68}$. Un processus similaire a eu lieu à Lyon et Aix-Marseille en 1973 sur les bases d'un séparatisme politique accusé.

Cette séparation des institutions sans séparation des corps s'est faite dans un contexte en rapide mutation sur le plan épistémologique : les années 1970-1971 virent

64 Période durant laquelle J. Dresch inonde ses instances de tutelle de lettres mettant l'accent sur le dénuement croissant de son établissement.

$\mathbf{6 5}$ C'est la thèse qui se dégage largement de l'ouvrage dirigé par Charles Soulié (2012).

$\mathbf{6 6}$ C'est J. Dresch qui, depuis son bureau de l'institut de géographie, a piloté la constitution du département de géographie de Vincennes, et même au-delà, comme en attestent les dizaines de courrier et de CV qu'il a reçus dans les derniers mois de 1968.

67 Où ne sont partis quasiment que des professeurs, plus quelques assistants de géomorphologie membres du PCF, d'où des problèmes structurels de recrutement.

68 À l'exclusion des cours de premier cycle, qui avaient déjà lieu ailleurs, à Censier avant 1968, dans divers centres à partir de 1970. 
le déploiement d'initiatives de rénovation qui, s'efforçant de dépasser les clivages politiques, entendaient concrétiser une modernisation de la géographie française, de plus en plus perçue comme sclérosée et en crise. Roger Brunet y joua un rôle de leader ${ }^{69}$, dans le lancement de la recherche collective sur programme " Les systèmes d'organisation de l'espace " (cf. Orain et Sol, 2007) et surtout dans la mise sur orbite de I'Espace géographique (dont la parution commença en 1972), revue appelée à concurrencer les Annales de géographie et à incarner un renouveau épistémologique souhaité par une proportion croissante de géographes. Alors que ces initiatives de rénovation étaient plutôt encouragées par la génération des A. Meynier, L. Papy, P. George ${ }^{70}$, etc., leur départ progressif à la retraite et une succession d'élections favorables aux autonomes étaient sur le point de laisser la place dans les comités (CNRS, CCU, CNFG) à des géographes au mieux favorables à une rénovation-préservation (J. Beaujeu-Garnier, P. Claval), sinon franchement hostiles à un questionnement de la doxa. Si la demande d'aide financière à l'édition de la nouvelle revue fut acceptée, bénéficiant des appuis importants de R. Brunet au CNRS, cette création apparait comme marquant la fin d'une période d'abondance (en moyens et en postes) à laquelle succéda une séquence beaucoup plus restrictive.

Au plan politique, 1972, année où est mis un terme à l'expérience de « nouvelle société » de Jacques Chaban-Delmas, annonce une reprise en main accrue de l'université renforçant en parallèle l'hégémonie de la droite universitaire et signant la fin des libéralités caractéristiques tant des années 1960 que des gages politiques donnés au " mouvement » dans la séquence 1968-1971"1. C'est aussi le moment où cessa le recrutement massif d'assistants, qui s'était poursuivi sinon intensifié entre 1968 et 1972. Il en résulta un sentiment de fermeture brutale sur fond de règlements de comptes.

\section{Années de plomb}

Pendant plus d'une dizaine d'années (1972-1984), la géographie française a vécu dans un climat fortement politisé et socialement clivé : des professeurs votant aux deux-tiers pour les listes des autonomes, des assistants et maîtres-assistants à proportion équivalente pour des syndicats de gauche; des joutes récurrentes autour des listes d'aptitude aux fonctions de maître-assistant; des campagnes électorales tendues, qui connurent un climax polémique dans les années suivant l'alternance de 1981; un sentiment récurrent de chasse aux sorcières frappant les équipes et les personnalités classées

69 En obtenant le ralliement de Paul Claval, il s'est associé à la fois une figure médiatrice des renouveaux anglo-américains et un représentant de la droite moderniste.

70 On retrouve une partie d'entre eux au comité de patronage de l'Espace géographique, aux côtés des modèles scientifiques de R. Brunet (É. Juillard, F. Taillefer, J. Tricart), d'économistes spatiaux (Jacques-René Boudeville, Claude Ponsard), de représentants fameux de la géographie théorique et quantitative anglo-scandinave (Brian Berry, Peter Gould, Peter Haggett, Törsten Hägerstrand) et de représentants ministériels et de figures de l'aménagement du territoire. 71 En exacte symétrie du tournant répressif qui frappa à la même époque les groupes gauchistes... 
à gauche ${ }^{72}$. La perspective défensive vantée par J. Bastié et ses alliés dans l'après-68 fut dans les faits vite convertie en position dominante dans les conseils administrant la discipline, confortée par un système majoritaire favorisant les cadres $\mathrm{A}$ et par des nominations ministérielles de plus en plus politico-syndicales (en particulier sous le long règne d'Alice Saunier-Seïté de 1976 à 1981). À bien des égards, cette période demeure encore à vif pour ses protagonistes et les éléments d'objectivation sont encore à construire afin de pouvoir dépasser un point de vue militant.

Pour autant, le rapport de force objectif fut atténué sur le plan symbolique par la posture sécessionniste de cette partie des nouvelles générations qui avait adhéré à Mai 68 et en avait gardé " l'humeur anti-institutionnelle " (Gobille, 2008a, 279), retraduite en termes disciplinaires dans une critique visant la "vieille géographie " incarnée par un CNFG honni's, les revues en place (Annales de géographie, BAGF, etc.), une organisation corporative ségrégationniste (ou chaque " rang " avait son association) et les messes communautaires (Journées géographiques). Au lieu d'investir le " système " et refusant la compromission, ils s'engagèrent massivement dans des expériences alternatives échappant au contrôle académique. Dans certains contextes locaux, une réticence analogue a pu se traduire par une forme de retrait disciplinaire : encouragés par un Bernard Kayser ${ }^{74}$ chantre de " la » science sociale, nombre d'assistants toulousains se sont concentrés sur des questions locales ou thématiques en se désinvestissant durablement de la scène géographique. Par contrecoup, il serait intéressant d'analyser le bénéfice qu'ont pu retirer d'une carrière orthodoxe ceux des géographes des nouvelles générations qui ont joué le jeu des institutions existantes et ont assuré la continuité de la tradition.

En définitive, plutôt que de privilégier un point de vue sur l'un ou l'autre " camp " qui se sont fait face - partiellement typés par le statut, l'ancrage générationnel, les valeurs scientifiques, etc. - , c'est la dimension agonistique elle-même que j'ai souhaité mettre en relief. Il est vraisemblable que l'on pourrait la tempérer en mettant à jour les liens locaux entre des " patrons » plus ou moins libéraux dans la réalité des relations interpersonnelles et des « assistants » que la socialisation professionnelle maintenait

72 Ce dont témoignent très fortement Michel Rochefort dans l'entretien déjà cité (Rochefort, 2002) mais aussi divers textes du début des années 1980. Ainsi l'introduction du colloque « Sens et non-sens de l'espace » (1984), intitulé « Témoignage d'une renaissance »: " Ce n'est pas le lieu de s'étendre ici, sinon pour les évoquer, sur les obstacles mis sur la route de ceux qui ne pensaient pas comme les représentants officiels de la discipline : carrières bloquées, projets avortés, mise à l'écart des instances décisionnelles. 》 (Burgel, Rochefort, Seronde-Babonaux, 1986, 9-10).

73 N'y appartenaient et n'y avaient droit de vote que les géographes-docteurs, qui devaient au demeurant être cooptés. Ce droit d'entrée sur fond de large œcuménisme est devenu un outil d'exclusion dans les années 1970, accompagnée de démissions fracassantes, achevant d'en faire un « club » (Bataillon, 2010).

74 Patron incontesté de la géographie humaine toulousaine durant plusieurs décennies, Bernard Kayser (1926-2001) a joué une partition assez spécifique en 1968, en position de go-between entre le mouvement contestataire et les autorités universitaires (homologue à celle d'autres élèves de P. George?). La séquence a plutôt conforté son autorité et son leadership, à l'abri de la critique. 
pour partie dans le "système ", via un régime de contrainte qui peut apparaître rétrospectivement relativement lâche. Celui-ci a pu les amener néanmoins à rechercher des affiliations moins compromettantes que les autres: CNRS ou Orstom plutôt qu'université, travaux « hors statut » dans des associations, recherche collective plutôt que thèse chef-d'œuvre, sans parler de tous ces chemins de traverse (enseignement, contrats d'aménagement, syndicalisme, expérience de luttes, etc.) qui ont pu retarder ou suspendre une épreuve académique (l'accomplissement d'une thèse d'État) à la fois symboliquement décriée et de rendement aléatoire dans le contexte d'incertitude des années 1970.

\section{La " nouvelle géographie ", une vocation d'hétérodoxie ?}

Perdure une interrogation essentielle, demeurée délibérément en marge de l'analyse jusqu'à présent : dans la séquentialité de la géographie française, le moment 1968 précède de très peu le début du processus de contestation puis de reconstruction disciplinaire que l'on désigne souvent par l'expression de "nouvelle géographie ». Nombre d'évocations historiques lient de façon automatique la crise politique générale et les mutations décisives qui se sont fait jour en géographie une poignée d’années après en utilisant la date comme un élément de périodisation (Marconnis, 1996), une contextualisation en gros (Scheibling, 1994) ou un passage obligé nécessitant un effort considérable de mise à distance (Claval, 1998). Au nom d'une conception sourcilleuse de l'autonomie de la science ou d'une défiance devant I'« illusion conséquencialiste » (Gobille, 2008a) prêtant à « 68 » toutes les responsabilités imaginables, cette association a pu être critiquée, mais là encore à un niveau très intuitif. Les lignes qui suivent sont un effort pour retracer les reconfigurations qui abondent ou nuancent l'idée d'une congruence entre la vocation critique des " années 68 » - visant l'autorité, la doxa, autrement dit les " grands partages " axiologiques, catégoriels ou sociaux, regardés comme allant de soi jusque-là, ou tout simplement l'existant, brutalement ramené à l'état de vieilleries - et ce qui s'est opéré en géographie dans les années suivantes ${ }^{75}$.

II convient de rappeler que la vogue de la «nouvelle géographie » est en premier lieu un mouvement inédit de critique de l'existant, faisant converger une critique épistémologique (la géographie produite en France jusque-là était préscientifique), politique (sous couvert d'apolitisme, elle servait les intérêts du pouvoir ou demeurait stérile) et méthodologique (le labourage encyclopédique d'un " terrain » doit céder la place à une problématisation commandant une procédure démonstrative). Le processus de remise en cause a très largement précédé la codification de manières de faire alternatives et suscité des initiatives d'ampleur et d'ambition diverses. La concomitance entre ce moment contestataire et la mise à disposition de méthodes quantitatives a conféré 
à ces dernières un potentiel révolutionnaire qu'elles n'avaient pas dans des disciplines voisines les ayant adoptées plus tôt (la trajectoire de la sociologie l'indique de façon exemplaire, voir Mauger, 1989). En revanche, le style épistémologique théorico-centré qu'elles apportaient éventuellement est apparu d'emblée comme attentatoire à la doxa empiriste de la géographie jusque-là prônée, et par là dotée d'un double potentiel de subversion et de relégitimation diversement apprécié. Certains, autour de la revue L'Espace géographique et de groupes informels apparus à l'issue de stages de formation aux techniques nouvelles, ont particulièrement exploré ce sillon théorico-quantitatif. D'autres, activistes déclarés au mitan des années 1970 autour des revues EspacesTemps (1975), Hérodote (1976) et Espaces et luttes (1978), ont pu se montrer plus circonspects à l'égard de conceptions jugées par trop " positivistes » et insuffisamment articulées à un horizon marxiste souvent jugé incontournable. Ces diverses sensibilités ont pu converger partiellement dans des lieux comme les colloques Géopoint (inaugurés en 1976) ou trouver des relais dans les syndicats de gauche. En revanche, à la différence de la contestation allemande quasi-contemporaine ${ }^{76}$, elles ne se sont pas appuyées sur des travaux savants opérant un réexamen critique systématique de la tradition géographique préexistante : il y va sans doute de l'absence d'un dispositif de type " habilitation » dans les cursus universitaires, qui aurait légitimé une telle entreprise, en regard de la lourdeur de la " thèse » française; il y va aussi surtout de la place très marginale et dévalorisée de l'historiographie dans la géographie de l'époque, encore (et pour longtemps) considérée comme superflue.

Une partie non négligeable des lycéens, étudiants et jeunes universitaires qui avaient « fait » ou regardé favorablement Mai 68 s'est ensuite investie vigoureusement dans l'expérience de rénovation de la discipline, même si la concordance n'est pas parfaite. De nombreux acteurs du mouvement théorico-quantitatif ont d'ailleurs souligné cette continuité, sans pouvoir toujours rationaliser l'articulation d'une séquence à l'autre, voire en la renvoyant à une contingence historique ${ }^{77}$. Dans cette perspective, il est tentant, à la suite de M. Pollak (1989) ${ }^{78}$, de lire l'expérience de l'échec politique comme le ressort d'un réinvestissement du motif critique dans un champ de pratique donnant davantage de prise sur l'ordre des choses, en l'occurrence une pratique disciplinaire, sujette dès lors à une opération de " subversion en pratiques " (Damamme et al., 2008) à une échelle réaliste. Cette reconversion critique ne s'est pas faite immédiatement, et rien ne l'atteste davantage que l'orthodoxie épistémologique encore

76 Voir la contribution de N. Ginsburger dans ce même numéro et son analyse des thèses d'habilitation de Gerard Hart et Dietrich Bartels.

77 Les entretiens réalisés par Sylvain Cuyala pour sa thèse Géo-histoire d'un mouvement scientifique. Les réseaux de la géographie théorique et quantitative européenne francophone (2014), donnent de nombreux exemples de réflexions autobiographiques sur l'incidence à la fois évidente et dérangeante de 68.

78 Voir l'analyse que j'en propose dans mon texte d'état de la question, "Une fertilisation paradoxale ? ", disponible dans le présent volume. 
de mise en mai-juillet 1968. II a fallu un déplacement partiel de la critique politique générale et sa retraduction sous la forme d'une critique disciplinaire spécifique, d'abord sous le diagnostic un peu vague d'une « crise » de la géographie (1970-1972). Ce dernier mobilisait un protagoniste connu (la géographie héritée) et un autre virtuel (la " nouvelle géographie » à faire). II avait de toute évidence la crise politique disciplinaire issue de 1968 pour horizon, doublée ou redimensionnée par la crise économique à partir du milieu des années 1970. II s'inspirait éventuellement de précédents devenus soudain mobilisables - I'un anglo-suédo-américain ${ }^{79}$ et dominant, l'autre soviétique et limité à une géographie physique systémiste ${ }^{80}$. Le besoin de renouvellement et de " bilan » qui s'est fait jour à l'occasion de cette « crise » - qui du point de vue épistémologique relevait initialement de la prophétie auto réalisatrice - n'a jamais trouvé d'expression aussi éloquente que dans les numéros de la première année de la revue L'Espace géographique (1972), lieu privilégié de figuration d'un schisme en cours. On y trouve d'ailleurs la seule figuration explicite d'une connexion avec la séquence politique précédente dans un article de R. Brunet annonçant (et de fait appelant de ses vœux) de « profondes transformations » épistémologiques :

Le " décollage » de ces nouvelles recherches a été favorisé par toute une série de contestations, dont beaucoup sont fort anciennes, mais qui ont pu converger, peut-être en partie grâce à ce catalyseur que furent les passionnants et interminables débats et remises en cause de 1968, et d'après. (Brunet, 1972)

Après avoir été annoncée par des analyses mesurées et délibérément surplombantes, la " crise ", jusque-là sporadique et réductible à des humeurs contestataires, s'est, à partir de 1973-1974, cristallisée et davantage configurée, produisant une dynamique critique que reflète une littérature contestataire abondante, de nouvelles revues au radicalisme affirmé, un séparatisme social prononcé, une volonté accrue de travail collectif en rupture avec le modèle de l'individu prenant en charge un territoire devenu sa spécialité, mais également un « style » dont l'air de famille avec 68 est troublant. La pratique du forum (comme collectif de discussion) y est omniprésente, et ne se réduit

79 La New Geography «théorique et quantitative » s'est développée aux États-Unis dès le milieu des années 1950 puis au Royaume-Uni, dans un climat dont la conflictualité est à réévaluer, acquérant en dix ans une audience et une assise institutionnelle prééminentes. Elle commence à être diffusée et traduite en France à un moment (le début des années 1970) où elle subit des critiques croissantes dans le monde anglophone, notamment sur son « positivisme » et sa neutralité politique, formulées en particulier par une Radical Geography qui aura beaucoup moins d'audience en France. En effet, ce qui ne fut pas temporellement associé dans la sphère anglophone (remise en cause épistémologique et critique politique) I'a en revanche été en France - ou en Allemagne, comme l'indique la contribution de Nicolas Ginsburger dans ce même volume.

80 Considérée par une poignée de géographes physiciens cherchant à développer des démarches " globales 》 ou intégrées (tels Georges Bertrand), dans un milieu où le PCF était solidement implanté et assez philosoviétique (comme en témoignent les cas de J. Tricart et J. Dresch), la géographie soviétique constituait néanmoins une pâle alternative au tropisme américain (et suédois). 
pas aux seules occurrences physiques, tels les colloques Géopoint ${ }^{81}$. On la retrouve aussi à l'écrit, tel cet article d'Y. Lacoste qui inaugure le premier numéro d'Hérodote (1976a) dans lequel le texte auctorial est discuté par un aréopage de « compagnons de route " qui le commentent dans les marges, grâce à un format (carré) qui permet une mise en page où les « notes » ont un espace abondant et moins minorisé que dans le dispositif infra-paginal habituel. II y va aussi d'un style polémique qui relaie et spécifie le " cadre global » (au sens de master frame, voir Gobille, 2008b) de la contestation soixante-huitarde des autorités instituées et en appelle à la tabula rasa. Ce style varie bien entendu fortement suivant les auteurs, du manifeste révolutionnaire aux accents " marxistes-léninistes » (mâtinés d'Althussser) qu'incarne le premier article de J. Lévy dans EspacesTemps (1975) ou de la rhétorique du dévoilement développée dans le " petit livre bleu » $d^{\prime} Y$. Lacoste (1976b) jusqu'aux prises de position plus intellectualisées d'un C. Raffestin (1976) ou au manifeste apaisé - mais néanmoins refondateur qui ouvre le premier Brouillon Dupont en 1977 (Chamussy et al., 1977).

Dernier trait que I'on mentionnera ici : corrélat de son humeur anti-institutionnelle, cette littérature contestataire de la deuxième moitié des années 1970 a reposé sur une économie précaire. À l'exception des revues dotées d'un éditeur (Doin pour L'Espace géographique, Maspero pour Hérodote), elle a utilisé des supports artisanaux, produisant ces « feuilles de chou » ronéotypées que furent les revues étudiantes Espaces Temps (à ses débuts) et Attila (qui publie un seul numéro en 1976), les Brouillons Dupont lancés en $1977^{82}$, Espaces et Luttes : minces volumes à la typographie aléatoire en format A4, faciles à reproduire, parfois retouchés à la main, brochés sommairement ${ }^{83}$, incorporant aisément des tracts, voire en mimant les dispositifs. L'illustration, ténue, renonce aux images traditionnellement indispensables à la géographie (cartes, coupes et blocs-diagrammes, photographies, etc.) et privilégie les petits dessins, caricatures ${ }^{84}$ et frises " décoratives ", exceptionnellement les bandes dessinées. Les schémas, le plus souvent " à flèches " (sagittaux), sont surtout prisés par les quantitativistes, pour lesquels ils sont davantage qu'une illustration : un temps du raisonnement, un mode d'élaboration alternatif au discours linéaire, même s'ils participent d'une reconfiguration de la géo-graphique. Du point de vue des genres textuels, la diversité et l'hétérogénéité prévalent : les articles programmatiques cohabitent avec

$\mathbf{8 1}$ Ils ont pour particularité de faire peu de place à des interventions orales individuelles, exception faite des conférences de " vedettes » du mouvement, et de privilégier les « ateliers », moments de débats entre congressistes.

82 II s'agit d'une revue liée au groupe éponyme, Dupont, créé en 1971 par de jeunes assistants qui suivaient des stages de géographie quantitative, plus tard co-organisateurs des colloques Géopoint, représentatifs de la sensibilité théoricoquantitative en cours de cristallisation en France, mais aussi fortement marqués à gauche pour la plupart.

$\mathbf{8 3}$ De ce point de vue, les volumes d'actes des colloques Géopoint ne sont pas foncièrement différents, même s'ils ont bénéficié de moyens humains et financiers plus conséquents.

84 Cette imagerie est en général bon enfant et n'a de l'esprit Hara Kiri/Charlie Hebdo et La Gueule ouverte (voir Guisnel, 2008) que la rapidité d'exécution et un fond caustique indexé sur l'esprit du temps. Dans Hérodote aussi, la caricature occupe une place importante, mais avec d'autres moyens, comme en témoignent des « portraits » de Michel Foucault ou François Châtelet assez élaborés figurant dans les premiers numéros. 
les textes d'humeur ou de controverse, les résultats empiriques pointus avec des bilans et enquêtes de toutes sortes et des comptes rendus d'actualité, sur fond d'adresse insistante à la communauté imaginée des lecteurs. En tout cela, cette production s'inscrit dans la mouvance plus générale de production de « fanzines de science sociale » dont Luc Boltanski a indiqué combien il avait été configurant pour les Actes de la recherche en sciences sociales ${ }^{85}$, et plus globalement dans un espace éditorial contre-culturel qui s'est amplement développé dans les années post-1968, sa fragilité étant la contrepartie d'une expression sans contraintes.

Au-delà de ces traits convergents, il conviendrait de souligner l'importante hétérogénéité des acteurs de cette contestation, tant dans ses pôles que dans la situation statutaire des individus concernés. Entre les petits groupes animés par les élèves de P. George (Y. Lacoste ${ }^{86}$, Raymond Guglielmo ${ }^{87}$ ), engagés dans des entreprises visant à approfondir le sens et la responsabilité politiques de la géographie (avec des objectifs assez différents à l'usage) et le gros des quantitativistes, les priorités et les modalités d'hétérodoxie divergent. L'ensemble a des propriétés démographiques typées mais peu typiques : très peu de "patrons ", ou alors en périphérie, assurant une insertion dans le mainstream; quelques maîtres assistants quadragénaires en cours de notabilisation; une masse d'assistants recrutés dans la période 1965-1972 et fortement féminisée (Pumain et Robic, 2002) ; quelques représentants des générations arrivées à l'université après 1968, menacés par la fermeture des recrutements après 1972, porteurs d'une radicalité renouvelée au mitan des années 1970 (groupes EspacesTemps et Attila). Pourtant, en l'absence d'une étude fine des itinéraires individuels, il est difficile de différencier socialement le gros des assistants ayant participé au mouvement de leurs homologues demeurés dans des trajectoires disciplinairement plus conformes (et auxquels ils ressemblent par ailleurs par l'attitude à l'égard des servitudes de la thèse d'État ou leur positionnement syndical). Dans le cas des théoriciens-quantitativistes, il semble surtout que des dynamiques locales ou régionales, propres à dessiner une

$\mathbf{8 5}$ Voir ce qu'en dit Marc Joly dans son article du présent volume, et les réflexions de Mathieu Quet sur la fondation contemporaine de Pandore.

86 Maître assistant à l'Institut de géographie de Paris, Yves Lacoste (né en 1929) rejoint Vincennes en 1969, auréolé par un précoce «Que sais-je? » sur le sous-développement publié en 1959. II développe alors de précieuses relations avec une partie de l'intelligentsia vincennoise (la « participationniste » et réformiste au principal, voir Soulié, 2012) à laquelle il sera dès lors associé, capital symbolique dont bénéficiera Hérodote après sa fondation en 1976. Producteur récurrent de manuels du secondaire, très inséré dans les milieux de l'« histégéo » (la discipline scolaire histoire-géographie, cet artefact français hautement idiosyncrasique), il a maintes fois affirmé avoir amorcé indépendamment de 68 une réflexion épistémologique stimulée par l'ennui suscité par la géographie académique de l'époque.

87 Bénéficiant lui aussi d'un statut de maître assistant en 1968 après un passage au CNRS, Raymond Guglielmo (19232011) a également rejoint Vincennes, après une expérience de Mai 68 vécue comme un révélateur et qui occasionne son départ du PCF (Guglielmo, 1991). Il y développe une " géographie des luttes » plus confidentielle parce que surtout préoccupée d'être au plus près des mouvements sociaux : Lip, Larzac, conflits liés à la désindustrialisation, etc. Il développe des collaborations avec l'économiste Michel Beaud, au sein de l'Association pour la critique des sciences économiques, et continue d'animer au sein du Laboratoire de géographie humaine de Paris 1 une équipe de géographie industrielle (sa spécialité académique). 
géographie différenciée du mouvement, rendent mieux compte de son éclosion ou de son refoulement que des marqueurs strictement sociaux ${ }^{88}$ : le mouvement est fort dans les universités de l'Est et du Sud-Est de la France peu dotées en patrons charismatiques ainsi que dans certains pôles plus récents ou isolés (Rouen), il est présent parmi d'autres à Paris, enfin inexistant là où il suscite de l'indifférence ou un consensus contre lui (grand Ouest, Toulouse, Bordeaux, mais aussi Vincennes).

De ce récit exploratoire des années 1968 des géographes français, mené en articulant des histoires locales et une perspective disciplinaire nationale, je voudrais retenir quelques éléments. Le plus immédiat concerne l'absence de synchronisation entre une temporalité politique et une temporalité scientifique des événements disciplinaires: si la première semble procéder par contrecoup assez rapide de la crise nationale, la seconde apparaît en décalage, après ce qui ressemble à une gestation relativement lente. On l'imagine abondée par de nombreux paramètres externes : un champ international dont l'influence devient ostensible et dans lequel les manières de faire de la géographie anglo-américaine donnent désormais le « la »; des marchés qui évoluent, dégageant des horizons professionnels dans l'expertise technique ou l'administration, alternatifs au métier de professeur ; une crise économique qui change la donne, etc. Ce léger déphasage n'a d'ailleurs rien d'étonnant, dès lors que l'on se souvient du caractère particulièrement routinier et légitimiste des élaborations " épistémologiques » de maijuillet 1968 : même s'il existait déjà des figures de la novation et de timides références à des alternatives à la doxa de l'école française, le travail critique n'a fait que débuter, et de façon fort graduelle, dans les premières années de la décennie 1970. De ce point de vue, 1968 apparait comme l'acte inaugural d'une ouverture des possibles, d'abord concédée par l'establishment géographique, très vite construite contre lui. Le contenu critique et les revendications d'aggiornamento, tout comme les nouvelles pratiques développées à la marge, différent de ce que l'on a pu connaître dans d'autres champs en raison exacte de ce qui pouvait être conçu comme hétérodoxe au regard des usages antérieurs. On comprend mieux ainsi le pouvoir de séduction d'une conception à la fois nomologique et politisée (en proportions variables), mais aussi davantage utile et configurée socialement, de la « nouvelle géographie » à inventer. Dans une époque où la passion de la " science " prend souvent des formes paradoxales et à fronts renversés - les pratiques littéraires se réclament de la science au moment où les scientifiques entreprennent sa critique (Gobille, 2005) -, les différentes sensibilités révolutionnaires de la géographie française partagent dans son invocation et sa mobilisation un étendard spécifique, quoique polymorphe et labile. 


\section{Bibliographie}

Allemand, S., 2007, Comment je suis devenu géographe?, Paris, Le Cavalier bleu.

Bataillon, C., 1981, « Table ronde imaginaire sur la géographie universitaire française 1930-1940 ", Hérodote, 20, p. 116-153.

Bataillon, C., 2006, « Six géographes en quête d'engagement : du communisme à l'aménagement du territoire. Essai sur une génération ", Cybergeo: European Journal of Geography, Épistémologie, Histoire de la Géographie, Didactique, document 341 (http://cybergeo. revues.org/1739, consulté le 9 janvier 2015).

Bataillon, C., 2010, Géographes. Génération 1930. À propos de Roger Brunet, Paul Claval, Olivier Dollfus, François Durand-Dastès, Armand Frémont et Fernand Verger, Rennes, Presses universitaires de Rennes (Espace et territoires).

Beaujeu-Garnier, J., 1971, La géographie : méthodes et perspectives, Paris, Masson (Collection de géographie appliquée).

Bennassar, B., 2000, " Mai 68 à l'université de Toulouse ", dans Demélas, M.-D., (dir.), Militantisme et histoire, Toulouse, Presses universitaires du Mirail (Tempus), p. 109-114.

Bisseret, N., 1968, "La sélection à l'université et sa signification pour l'étude des rapports de dominance ", Revue française de sociologie, IX/4. p. 463-496.

Brunet, R., 1972, " Les nouveaux aspects de la recherche géographique : rupture ou raffinement de la tradition?", L'Espace géographique, I/2, p. 73-77.

Burgel, G., Rochefort, M., SerondeBabonaux, A.-M., 1984, "Témoignage d'une renaissance ", dans Collectif français de géographie urbaine et sociale, De la géographie urbaine à la géographie sociale. Sens et non-sens de l'espace, Paris, p. 9-10.

Callu, A. (dir.), 2010, Le Mai 68 des historiens : entre identités narratives et histoire orale, Villeneuve d'Ascq, Presses universitaires du Septentrion.
Chamussy, H., Charre, J., Durand, M.-F., Le Berre, M., 1977, " Espace, que de brouillons commet-on en ton nom! ", Brouillons Dupont, 1, p. 15-30.

Claval, P., 1996, " La vie universitaire : avant, pendant et après 1968 ", dans La géographie comme genre de vie. Un itinéraire intellectuel, Paris, L'Harmattan (Géographies en liberté), p. 59-64.

Claval, P., 1998, "Mai-1968 » et « Chapitre 11, La géographie en mutation : avant et après 1968. ", dans Histoire de la géographie de 1870 à nos jours, Paris, Nathan Université (références), p. 302-305 et p. 323-336.

Collectif, 1968, "Les États généraux de la géographie. Tours, 8-10 juillet. Procès-verbaux, rapports, motions ", Intergéo, 12, p. 177-217.

Daudel, C., 2008, Jean Demangeot géographe de terrain, Paris, L'Harmattan (Patrimoine des géographes).

Dosse, F., 2008, " Vincennes (19691974) : entre science et utopies », dans Artières, P., Zancarini-Fournel, M. (dir.), 68: Une histoire collective, 1962-1981, Paris, La Découverte, p. 505-513.

Gaubert, C., Pouly, M.-P., 2012, "Transformations morphologiques et mobilisations disciplinaires. Les enseignants et étudiants de I'Institut d'anglais de la Sorbonne en 1968 ", Actes de la recherche en sciences sociales, 194, p. 78-97.

Gobille, B., 2005 (mai), " La guerre de Change contre la "dictature structuraliste" de Tel Quel. Le "théoricisme" des avant-gardes littéraires à l'épreuve de la crise politique de Mai 68 ", Raisons politiques, 18, p. 73-96.

Gobille, B., 2008a, "La vocation d'hétérodoxie ", dans Damamme, D. et al., Mai Juin 68, Paris, Éditions de l'Atelier, p. 274-291.

Gobille, B., 2008b, "L'événement Mai 68. Pour une sociohistoire du temps court", Annales. Histoire, sciences sociales, 2008/2, 63e année, p. 321-349. 
Guglielmo, R., 1991, "Les années 70 : une recherche engagée et contestataire ", dans Moulin, B., Plet, F. (dir.), Autour de Raymond Guglielmo. Géographie et contestations. Centre de recherche sur les espaces de vie, SaintDenis, CREV/Université Paris VIII, p. 5-11.

Guisnel, J., 2008, "La nouvelle presse : de Hara-Kiri à Libération ", dans Artières, P., Zancarini-Fournel, M. (dir.), 68: Une histoire collective, 1962-1981, Paris, La Découverte, p. 600-606.

Lacoste, Y., 1976a, " Pourquoi Hérodote? Crise de la géographie et géographie de la crise ", Hérodote, 1, p. 9-62.

Lacoste, Y., 1976b, La géographie, ça sert d'abord à faire la guerre, Paris, François Maspero.

Lévy, J., 1975, " Pour une géographie scientifique », EspacesTemps, 1, p. 46-65.

Marconis, R., 1996, Introduction à la géographie, Paris, Armand Colin (U), rééd. 2000.

Mauger, G., 1989 (avril), « L'approche biographique en sociologie : une démarche "contestataire", Cahiers de I'IHTP, " Mai 68 et les sciences sociales ", 11, p. 85-99.

Meynier, A., 1969, Histoire de la pensée géographique en France (1872-1969), Paris, PUF.

Moulin, B., Plet, F. (coord.), 1991, Autour de Raymond Guglielmo. Géographie et contestations, Saint-Denis, CREV.

Orain, 0., 2009a, De plain-pied dans le monde. Écriture et réalisme dans la géographie française au xxe siècle, Paris, L'Harmattan (Histoire des sciences humaines).

Orain, 0., 2009b (septembre), « Écrire sur 68 en spécialiste, tournant ou accomplissement? ", Genèses, 76, p. 137-156.

Orain, O., Sol, M.-P., 2007 (automne), " Les géographes et le travail collectif. La recherche coopérative sur programme à l'œuvre ", La Revue pour I'histoire du CNRS, 18, p. 11-14.
Prost, A., 1989, « 1968 : mort et naissance de l'université française ", Vingtième Siècle, 23, p. 59-70 [repris dans Éducation, société et politiques. Une histoire de l'enseignement en France, de 1945 à nos jours, Paris, Seuil (Points histoire), 1992, rééd. Seuil (xxe siècle), 1997].

Prost, A., 2004, Histoire générale de l'enseignement et de l'éducation en France. IV. L'École et la famille dans une société en mutation (depuis 1930), Paris, Perrin (Tempus), ici p. 311-412.

Pumain, D., Robic, M.-C., 2002 (avril), " Le rôle des mathématiques dans une "révolution" théorique et quantitative : la géographie française depuis les années $1970 "$ ", Revue $d^{\prime}$ histoire des sciences humaines, 6, p. 123-144.

Raffestin, C., 1976, " Problématique et explication en géographie humaine ", Géopoint 76, Théories et géographie, p. 81-96.

Rebeyrol, Y., 1968, "Les Géographes à la recherche de la géographie ", Le Monde, 13 juillet 1968, p. 10.

Robic, M.-C., 1989 (mai), « Un siècle de professionnalisation ", dans Comité national d'évaluation, La géographie dans les universités françaises. Une évaluation thématique. Rapport d'évaluation, Paris, p. 17-19, 31-33 et 199-210.

Robic, M.-C. et al., 2006, Couvrir le monde. Un grand $x x^{e}$ siècle de géographie française, Paris, ADPF/La Documentation française.

Rochefort, M., 2002, " Mai 68 : ruptures à l'institut de Géographie. Entretien avec Nicole Mathieu et Stéphane Cartier ", dans Paix, C. (dir.), Parcours dans la recherche urbaine. Michel Rochefort, un géographe engagé, Strates, hors-série, p. 239-254.

Saunier-Seïté, A., 1982, En première ligne. De la communale aux universités, Paris, Plon.

Scheibling, J., 1994, Qu'est-ce que la géographie?, Paris, Hachette (Carré Géographie). 
RHSH 26 - Les " années 68 " des sciences humaines et sociales

Soulié, C. (dir.), 2012, Un mythe à détruire? Origines et destin du centre universitaire expérimental de Vincennes, Saint-Denis, Presses universitaires de Vincennes.
Tissier, J.-L., 1985, « Les anciens élèves de l'École normale de Saint-Cloud et la géographie française, 1942-1973", dans Charle, C., Ferré, R. (dir.), Le Personnel de l'enseignement supérieur en France aux xix et $x x^{e}$ siècles, Paris, CNRS Éditions, p. 205-218. 
Olivier Orain • Mai 68 et ses suites en géographie française

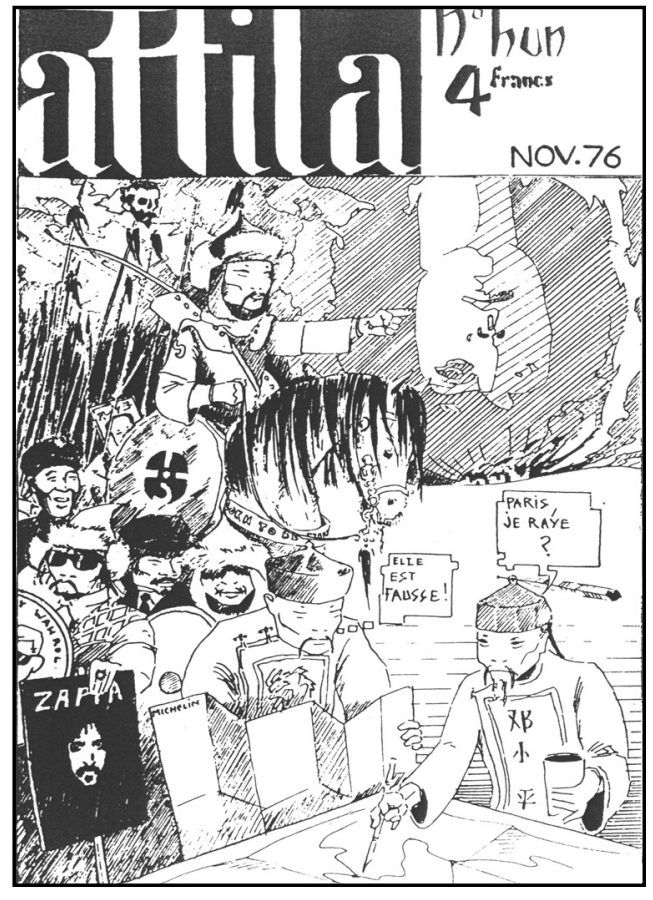

Fig. 1 - Page de couverture du premier (et unique) numéro de la revue d'étudiants Attila (1976) [Collection bibliothèque Épistémologie et histoire de la géographie, Paris].
Fig. 2 - Page de garde du premier numéro des Brouillons Dupont (1977) [Collection bibliothèque Épistémologie et histoire de la géographie, Paris].

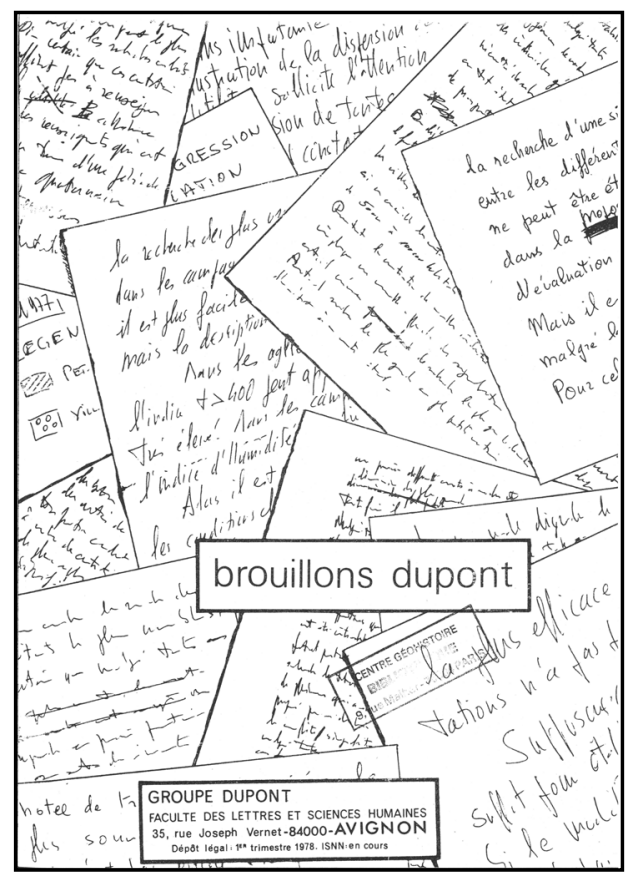


RHSH 26 - Les " années 68 " des sciences humaines et sociales

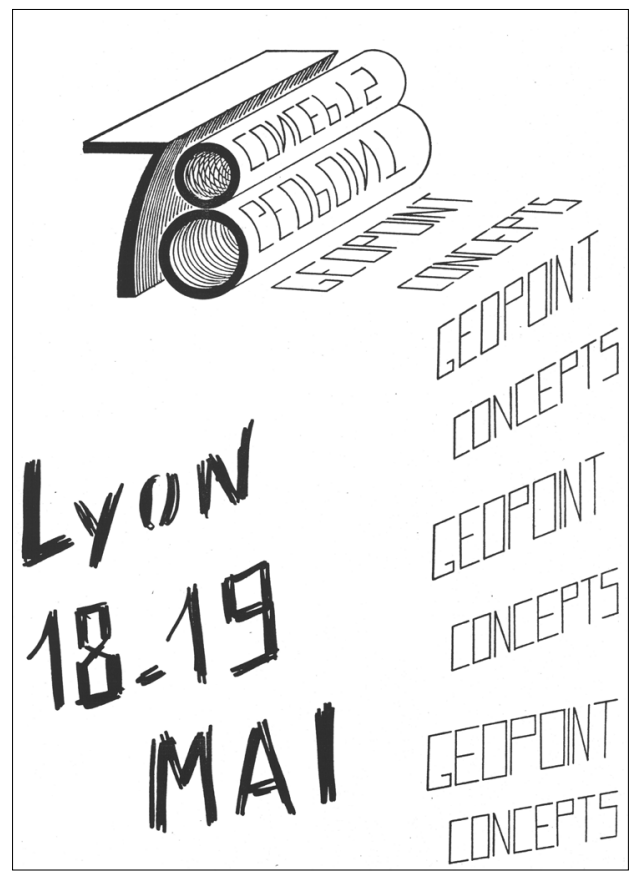

Fig. 3 - Annonce du colloque Géopoint dans le $\mathrm{n}^{\circ} 2$ des Brouillons Dupont (1978) [Collection bibliothèque Épistémologie et histoire de la géographie, Paris].

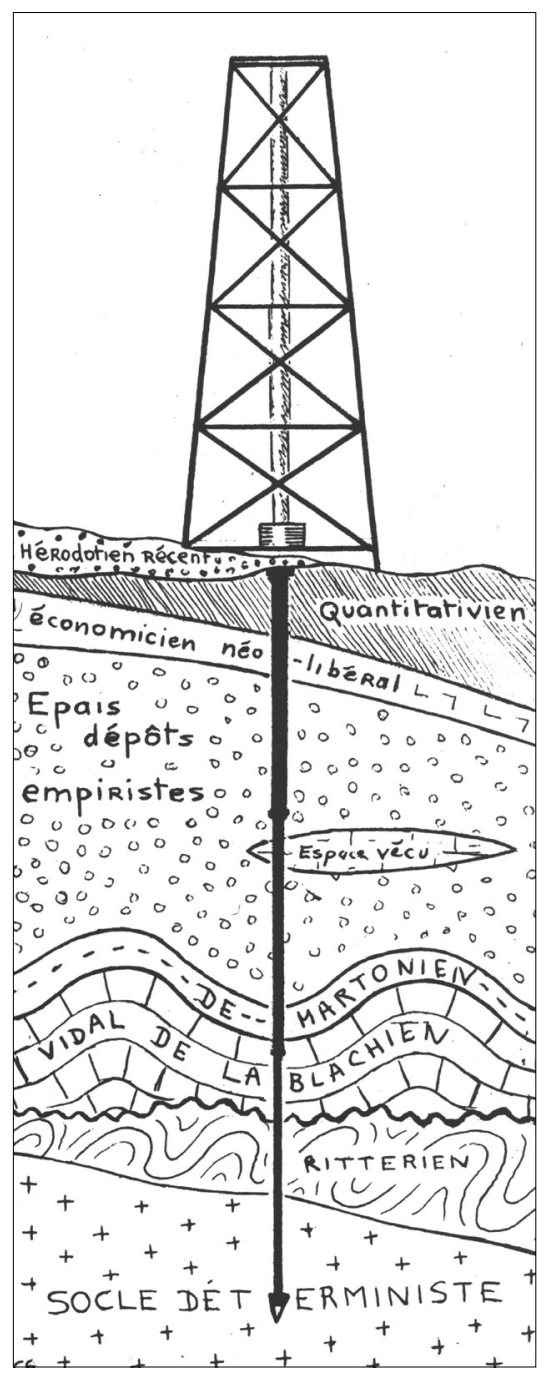

Fig. 4- Illustration de l'article de Christian Grataloup, " La géographie en réponses », EspacesTemps, n 4, 1976 [C. Grataloup]. 